\title{
RELAXATION OF CONVEX FUNCTIONALS: THE GAP PROBLEM
}

\section{RELAXATION DE FONCTIONNELLES CONVEXES: LE PROBLÈME DU GAP}

\author{
E. ACERBI ${ }^{\mathrm{a}}$, G. BOUCHITTÉ ${ }^{\mathrm{b}}$, I. FONSECA $^{\mathrm{c}, *}$ \\ a Dipartimento di Matematica, Via Massimo D’Azeglio 85/A, 43100 Parma, Italy \\ ${ }^{\mathrm{b}}$ Département de mathématiques, Université de Toulon et du Var, BP 132, \\ 83957 La Garde Cedex, France \\ ${ }^{\mathrm{c}}$ Department of Mathematical Sciences, Carnegie Mellon University, Pittsburgh, PA, USA
}

Received 17 July 2001, revised 18 January 2002

ABSTRACT. - It is shown that the relaxed energy

$$
\mathcal{F}(u, A):=\inf \left\{\liminf _{n \rightarrow+\infty} \int_{A} f\left(x, \nabla u_{n}\right) d x:\left\{u_{n}\right\} \subset W_{\text {loc }}^{1, \beta}\left(A ; \mathbb{R}^{d}\right), u_{n} \rightarrow u \text { in } L^{1}\left(A ; \mathbb{R}^{d}\right)\right\},
$$

admits the representation

$$
\mathcal{F}(u, A)=\int_{A} f(x, \nabla u) d x+\mu_{s}(u, A),
$$

where $f$ is a convex, Carathéodory integrand satisfying a nonstandard " $\alpha-\beta$ " growth hypothesis, $\beta \in[\alpha, N \alpha /(N-1))$. Sufficient conditions guaranteeing that $\mu_{s}(u, \cdot)=0$ are discussed. An example asserting that this representation may fail in the quasiconvex case is provided.

\footnotetext{
* Corresponding author.

E-mail addresses: acerbi@prmat.math.unipr.it (E. Acerbi), bouchitte@univ-tln.fr (G. Bouchitté), fonseca@andrew.cmu.edu (I. Fonseca).
} 
(C) 2003 L'Association Publications de l'Institut Henri Poincaré. Published by Elsevier B.V. All rights reserved MSC: 49J45; 74B20

Keywords: Convexity; Besicovich Covering Theorem; Radon Nikodym derivative; Lavrentiev phenomenon

RÉSUMÉ. - Nous montrons que l'énergie relaxée

$$
\mathcal{F}(u, A):=\inf \left\{\liminf _{n \rightarrow+\infty} \int_{A} f\left(x, \nabla u_{n}\right) d x:\left\{u_{n}\right\} \subset W_{\text {loc }}^{1, \beta}\left(A ; \mathbb{R}^{d}\right), u_{n} \rightarrow u \text { in } L^{1}\left(A ; \mathbb{R}^{d}\right)\right\},
$$

admet la représentation intégrale

$$
\mathcal{F}(u, A)=\int_{A} f(x, \nabla u) d x+\mu_{s}(u, A),
$$

lorsque $f$ est un intégrande convexe de Carathéodory vérifiant une condition de croissance non standard de type " $\alpha-\beta$ " avec $\beta \in[\alpha, N \alpha /(N-1))$. Des conditions suffisantes assurant que $\mu_{s}(u, \cdot)=0$ sont proposées ainsi qu'un exemple montrant que la représentation obtenue ne s'applique pas au cas quasiconvexe.

(C) 2003 L'Association Publications de l'Institut Henri Poincaré. Published by Elsevier B.V. All rights reserved

\section{Introduction}

The Lavrentiev phenomenon, or gap problem, has stirred renewed interest in recent years as it challenges traditional theories in the Calculus of Variations. A prototype model, relevant to the study of cavitation in rubber-like materials (see $[3,20,27,28]$, among others), assigns to each deformation $u \in W^{1, N}\left(\Omega ; \mathbb{R}^{N}\right)$ the total energy

$$
F(u, \Omega):=\int_{\Omega}|\nabla u|^{p}+|\operatorname{det} \nabla u| d x,
$$

where $\Omega$ is an open, bounded domain in $\mathbb{R}^{N}$, and $p \in(N-1, N)$. Clearly sequences of deformations in $W^{1, N}\left(\Omega ; \mathbb{R}^{N}\right)$ with bounded energy will be weakly compact in $W^{1, p}\left(\Omega ; \mathbb{R}^{N}\right)$ but not necessarily in $W^{1, N}$, so it may be possible to approach energetically functions $u \in W^{1, p}\left(\Omega ; \mathbb{R}^{N}\right) \backslash W^{1, N}\left(\Omega ; \mathbb{R}^{N}\right)$. We then seek to characterize the limiting, effective energy associated to $u$. This example has been studied at length, and in particular we refer to $[1,9,16]$. More generally, consider a bulk energy density $f: \Omega \times \mathbb{R}^{d \times N} \rightarrow[0,+\infty)$ satisfying the following standing hypotheses:

(H1) $f$ is Carathéodory;

(H3) [“ $\alpha-\beta$ "growth condition] $|z|^{\alpha} \leqslant f(x, z) \leqslant C\left(1+|z|^{\beta}\right)$ for all $z \in \mathbb{R}^{d \times N}, \mathcal{L}^{N}$ a.e. $x \in \Omega$, and for some $C>0$, where $1<\alpha \leqslant \beta<\frac{N \alpha}{N-1}$.

For every open set $A \subset \Omega$ and every $u \in W_{\text {loc }}^{1,1}\left(A ; \mathbb{R}^{d}\right)$ we set

$$
F(u, A):=\int_{A} f(x, \nabla u(x)) d x,
$$


and for every $u \in L^{1}\left(A ; \mathbb{R}^{d}\right)$ we introduce the relaxed functional

$$
\mathcal{F}(u, A):=\inf \left\{\liminf _{n \rightarrow+\infty} F\left(u_{n}, A\right):\left\{u_{n}\right\} \subset W_{\text {loc }}^{1, \beta}(A), u_{n} \rightarrow u \text { in } L^{1}\left(A ; \mathbb{R}^{d}\right)\right\} .
$$

We search for an integral representation for $\mathcal{F}(u, A)$.

When $\alpha=\beta$ and (H1), (H3) hold, then it is well known that (see $[2,3,10,26])$

$$
\mathcal{F}(u, A)=\int_{A} Q f(x, \nabla u) d x,
$$

where the quasiconvex envelope $Q f$ of $f$ is defined by

$$
Q f(\xi):=\inf \left\{\int_{(0,1)^{N}} f(\xi+\nabla \varphi(x)) d x: \varphi \in W_{0}^{1, \infty}\left(Q ; \mathbb{R}^{d}\right)\right\} .
$$

If $\alpha<\beta-\beta / N$ then one may have $\mathcal{F}(u, \Omega)=0$ (see [4]), and in the case where $\alpha=\beta-\beta / N$ it may happen that $\mathcal{F}(u, \cdot)$ is not even subadditive (see [11]). When $f$ does not depend on the position vector $x \in \Omega$ these degeneracies cannot occur if $\beta \in[\alpha, \alpha N /(N-1))$. Within this range, and using the global method for relaxation introduced by Bouchitté, Fonseca and Mascarenhas (see [7]), together with an extension operator from $W^{1, p}$ into $W^{1, q}$ obtained by Fonseca and Malý (see [15], Lemma 2.2), it was proven in [6], Theorem 3.1, that if $f=f(\nabla u)$ satisfies (H3) and if $\mathcal{F}(u, \Omega)<+\infty$ then

$$
\mathcal{F}(u, A)=\int_{A} Q f(\nabla u) d x+\mu_{s}(u, A)
$$

for all open sets $A \subset \Omega$ and for some finite, Radon measure $\mu_{s}(u, A)$, singular with respect to $\mathcal{L}^{N}\llcorner\Omega$, the $N$-dimensional Lebesgue measure in $\Omega$. Earlier results on lower semicontinuity for certain ranges $\alpha<\beta$ and with quasiconvex integrands were obtained by [24,25], and for polyconvex energy densities with $\alpha \geqslant N-1, \beta=N$, we refer to $[1,8,9,11,12,17,18,21-23]$.

In this paper we address the effect of considering an inhomogeneous density, i.e. $f=f(x, \xi)$. In the main theorem of this paper we show that the analogue to (1.2),

$$
\mathcal{F}(u, A)=\int_{A} Q f(x, \nabla u) d x+\mu_{s}(u, A),
$$

still holds provided $f$ satisfies $(\mathrm{H} 1),(\mathrm{H} 3)$ and

(H2) $f(x, \cdot)$ is convex for $\mathcal{L}^{N}$ a.e. $x \in \Omega$.

Precisely, we prove

THEOREM 1.1. - If (H1)-(H3) hold, if $A$ is an open subset of $\Omega$, if $u \in L^{1}\left(A ; \mathbb{R}^{d}\right)$, and if $\mathcal{F}(u, A)<+\infty$, then for every open set $B \subset A$

$$
\mathcal{F}(u, B)=\int_{B} f(x, \nabla u) d x+\mu_{s}(u, B)
$$


where $\mu_{s}(u, \cdot)$ is a nonnegative Radon measure singular with respect to $\mathcal{L}^{N}$.

Again the proof of this result is strongly hinged on the global method of relaxation introduced in [7] (see also [5]), although some of the arguments may now be greatly simplified by exploiting the convexity assumption. In particular, the uplifting operator $\mathcal{P}$ introduced in [15] (see also [6]) is no longer needed. The proof presented in Section 3 concerns the scalar case where $d=1$ and the argument is entirely similar in the vector valued-scalar case. Therefore and for simplicity, we leave the obvious adaptations to the reader.

Section 4 is devoted to the study of several sufficient conditions ensuring that the Lavrentiev phenomenon does not occur. In view of Ioffe's Theorem (see Theorem 2.4), the functional $F$ is lower semicontinuous and thus $F \leqslant \mathcal{F}$. We then say that the Lavrentiev phenomenon occurs when at some point we have the strict inequality $F<\mathcal{F}$.

The first result, deduced directly from Theorem 1.1, asserts that the Lavrentiev phenomenon is local, precisely,

Proposition 1.2. - Let $A$ be an open subset of $\Omega$ and let $u \in L^{1}(A)$ with $\mathcal{F}(u, A)<$ $+\infty$. Assume that for every $x \in A$ there is an open neighbourhood $U$ of $x$ such that

$$
\mathcal{F}(u, U)=F(u, U) .
$$

Then $\mathcal{F}(u, B)=F(u, B)$ for every open set $B \subset A$.

An interesting model example of convex functionals depending on $x$ and with a growth-coercivity gap as in (H3) is provided by $\int|D u|^{p(x)} d x$ for some function $p: \Omega \rightarrow \mathbb{R}$ (see $[29,30]$ ), or, more generally,

$$
u \mapsto \int_{\Omega} f(x, \nabla u(x)) d x
$$

where $f: \Omega \times \mathbb{R}^{N} \rightarrow[0,+\infty)$ satisfies

$$
|z|^{p(x)} \leqslant f(x, z) \leqslant C\left(1+|z|^{p(x)}\right) \quad \text { for all } z \in \mathbb{R}^{N} \text {, a.e. } x \in \Omega,
$$

and for some $C>0$. The proof of Theorem 1.3 below follows closely the original argument of Zhikov and Fan (see [29]), although some of the technical difficulties encountered by those authors may now be avoided using the theory developed in this paper.

Let $p: \Omega \mapsto \mathbb{R}$ be a continuous function such that

$$
1<\alpha \leqslant p(x) \leqslant \beta
$$

and

$$
|p(x)-p(y)| \leqslant \frac{\gamma}{|\log | x-y||} \quad \text { whenever } 0<|x-y| \leqslant \frac{1}{2},
$$

for some $\alpha, \beta, \gamma>0$. 
THEOREM 1.3. - Let $f: \Omega \times \mathbb{R}^{N} \rightarrow[0,+\infty)$ satisfy (H1), (H2), (1.5), and assume that (1.6) holds. If $u \in W_{\mathrm{loc}}^{1, \alpha}(\Omega)$ is such that $f(\cdot, \nabla u) \in L_{\mathrm{loc}}^{1}(\Omega)$ then

$$
\mathcal{F}(u, \cdot)=F(u, \cdot) .
$$

The last section of this work, Section 5, is dedicated in its entirety to the treatment of an example falling into the class of prototype energies (1.1) which satisfy (H1), (H3), are polyconvex rather than convex, and for which a genuine measurable dependence on $x$ prevents (1.3) to hold. This fact provides one more evidence that quasiconvex energies and convex energies do share quite different properties, and that care must be taken when generalizing results from the convex to the non-convex setting. With Proposition 1.4 we show that Theorem 1.1 may be false if stated for integrands $f: \Omega \times \mathbb{R}^{N} \times \mathbb{R}^{d \times N} \rightarrow$ $[0,+\infty), d, N>1$, satisfying (H1), (H3), and

$\left(\mathrm{H} 2^{\prime}\right) f(x, \cdot)$ is quasiconvex for $\mathcal{L}^{N}$ a.e. $x \in \Omega$.

We recall that a Borel function $g: \mathbb{R}^{d \times N} \rightarrow \mathbb{R}$ is said to be quasiconvex (see [Morrey]) if

$$
\int_{(0,1)^{N}} g(\xi+\nabla \varphi(y)) d y \geqslant g(\xi)
$$

for all $\xi \in \mathbb{R}^{d \times N}$ and all $\varphi \in W_{0}^{1, \infty}\left((0,1)^{N} ; \mathbb{R}^{d}\right)$. Clearly convex functions are quasiconvex, and a simple example of a quasiconvex function which is not convex is given by $\xi \mapsto|\operatorname{det} \xi|$ (for a detailed study of quasiconvexity we refer the reader to $[1,2,9,11$, $12,16,17,23-26])$. In the absence of growth-coercivity gap, i.e. when $\alpha=\beta$ in (H3), it was proven by Acerbi and Fusco (see [2]), that $F(\cdot, \Omega)$ is sequentially weakly lower semicontinuous in $W^{1, \alpha}\left(\Omega ; \mathbb{R}^{d}\right)$, and, in addition, it can be shown that (see also [10])

$$
\mathcal{F}(u, \Omega)=\int_{\Omega} f(x, \nabla u(x)) d x .
$$

If there is a gap and under (H1), (H3), then Theorem 3.1 in [15] still holds, and so for $u \in W^{1, \alpha}\left(\Omega ; \mathbb{R}^{d}\right)$ such that $\mathcal{F}(u, \Omega)<+\infty$ we have

$$
\mathcal{F}(u, A)=\int_{A} f^{u}(x) d x+\mu_{s}(u, A) \quad \text { for all } A \text { open subset of } \Omega,
$$

where $\mu_{s}(u, \cdot)$ is a nonnegative finite Radon measure on $\Omega$, singular with respect to $\mathcal{L}^{N}\left\llcorner\Omega\right.$, and for some density $f^{u}$.

The next natural question is: under which conditions can we guarantee that

$$
f^{u}(x)=f(x, \nabla u(x)) \quad \text { for a.e. } x \in \Omega ?
$$

Theorem 1.1 tells us that (H2), i.e. the convexity of $f(x, \cdot)$ for a.e. $x \in \Omega$, implies the identity in (1.7), and Theorem 3.2 in [6] ensures (1.7) if ( $\left.\mathrm{H}^{\prime}\right)$ holds and if $f$ does not depend on $x$. 
Here we show that this last statement is close to being optimal, in that if $f$ has a genuine measurable dependence on $x$, and even though $(\mathrm{H} 1),\left(\mathrm{H} 2^{\prime}\right),(\mathrm{H} 3)$ still hold, then it may happen that

$$
f^{u}(x)<f(x, \nabla u(x)) \text { for all } x \in E,
$$

where $E$ is some set with $|E|>0$. The construction of such function $f$ is motivated by the result of Gangbo (see [18], Theorem 3.1) asserting that

$$
u \in W^{1, N}\left(\Omega ; \mathbb{R}^{N}\right) \mapsto \int_{\Omega} \chi_{K}(x)|\operatorname{det}(\nabla u(x))| d x
$$

is lower semicontinuous in $W^{1, N}\left(\Omega ; \mathbb{R}^{N}\right)$ for the weak topology of $W^{1, p}\left(\Omega ; \mathbb{R}^{N}\right)$, with $N-1<p<N$, if and only if $|\partial K|=0$.

Proposition 1.4. - Fix $\theta \in(0,1)$, let $p \in(N-1, N)$, let $\Omega \subset \mathbb{R}^{N}$ be an open, bounded domain, let $K$ be a compact subset of $\Omega$ with $|\partial K|>0$, and let $f: \Omega \times$ $\mathbb{R}^{N \times N} \rightarrow[0,+\infty)$ be given by

$$
f(x, \xi):=\theta|\xi|^{p}+\chi_{K}|\operatorname{det} \xi| \text { for a.e. } x \in \Omega \text {, and all } \xi \in \mathbb{R}^{N \times N} .
$$

If $\operatorname{id}(\cdot)$ is the identity function and $\mathbb{I}$ is the identity matrix, then there exists $\theta_{0} \in(0,1)$ such that if $\theta<\theta_{0}$ then for all $x \in \partial K$

$$
f^{\mathrm{id}}(x)<f(x, \mathbb{I}) .
$$

It is clear that $f$ satisfies (H1), (H3) with $\alpha:=p, \beta:=N$, and $f(x, \cdot)$ is quasiconvex. In light of Proposition 1.4, we have

$$
\mathcal{F}(\mathrm{id}, A)=\int_{A} f^{\mathrm{id}}(x) d x+\mu_{s}(u, A),
$$

where there holds, for $\theta$ sufficiently small, $f^{\text {id }}(x)<f(x, \mathbb{I})$ for all $x \in \partial K$.

\section{Preliminaries}

Let $\Omega$ be an open, bounded subset of $\mathbb{R}^{N}$, and consider a function $f: \Omega \times \mathbb{R}^{N} \rightarrow$ $[0,+\infty)$ satisfying the following standing hypotheses:

(H1) $f$ is Carathéodory;

(H2) $f(x, \cdot)$ is convex for $\mathcal{L}^{N}$ a.e. $x \in \Omega$;

(H3) $|z|^{\alpha} \leqslant f(x, z) \leqslant C\left(1+|z|^{\beta}\right)$ for all $z \in \mathbb{R}^{N}, \mathcal{L}^{N}$ a.e. $x \in \Omega$, and for some $C>0$, where

$$
1<\alpha \leqslant \beta<\frac{N \alpha}{N-1} \text {. }
$$

We recall that $f$ is said to be Carathéodory if $f(\cdot, z)$ is $\mathcal{L}^{N}$-measurable for all $z$ and $f(x, \cdot)$ is continuous for $\mathcal{L}^{N}$ a.e. $x \in \Omega$, where $\mathcal{L}^{N}$ stands for the $N$-dimensional 
Lebesgue measure on $\mathbb{R}^{N}$. Often we will write $|E|$ in place of $\mathcal{L}^{N}(E)$. Also, throughout this paper the letter $C$ will denote a generic positive constant which may vary from line to line and within the same formula. Note that in view of (2.1), if $A \subset \mathbb{R}^{N}$ is an open set with Lipschitz boundary then the inclusion $W^{1, \alpha}(\partial A) \subset W^{1-1 / \beta, \beta}(\partial A)$ is continuous and compact, where $W^{1-1 / \beta, \beta}(\partial A)$ is the space of traces on $\partial A$ of functions in $W^{1, \beta}(A)$. More generally standard results in the theory of Sobolev spaces yield the lifting property presented below.

LEMmA 2.1. - If $A \subset \mathbb{R}^{N}$ is an open, bounded, Lipschitz domain and if (2.1) holds, then there exists a continuous, linear, compact mapping

$$
\mathrm{E}_{A}: W^{1, \alpha}(\partial A) \rightarrow W^{1, \beta}(A) \text { such that } \operatorname{tr} \mathrm{E}_{A}(g)=g \text { on } \partial A .
$$

Remark 2.2. - Since the "uplift" operator $\mathrm{E}_{A}$ of Lemma 2.1 is constructed with symmetric convolution kernels, it is readily verified that affine functions remain unchanged, i.e., if $u(x)=a+b \cdot x$ then $\mathrm{E}_{A} u=u$.

Remark 2.3. - (i) The growth hypothesis (H3) together with the convexity of $f$ imply that $f$ is $\beta$-locally Lipschitz continuous with respect to $z$, i.e., for a.e. $x \in \Omega$ and all $z, w \in \mathbb{R}^{N}$ (see $\left.[10,24,25]\right)$

$$
|f(x, z)-f(x, w)| \leqslant C\left(1+|z|^{\beta-1}+|w|^{\beta-1}\right)|z-w| .
$$

(ii) Let $\left\{z_{i}\right\}$ be a countable dense subset of $\mathbb{R}^{N}$, and for all $i$ let $K_{i}$ be the set of Lebesgue points of the function $f\left(\cdot, z_{i}\right)$. Setting $\mathbb{K}(f):=\bigcap K_{i}$, then $|\Omega \backslash \mathbb{K}(f)|=0$, and if $x_{0} \in \mathbb{K}(f)$ then it is a Lebesgue point for $f(\cdot, z)$ for all $z \in \mathbb{R}^{N}$. Indeed, fix $0<\varepsilon<1$, let $M:=1+|z|$, and choose $z_{i}$ with $\left|z-z_{i}\right|<\varepsilon$. By (2.2)

$$
\left|f(x, z)-f\left(x_{0}, z\right)\right| \leqslant C M^{\beta-1}\left|z-z_{i}\right|+\left|f\left(x, z_{i}\right)-f\left(x_{0}, z_{i}\right)\right|,
$$

thus for $\rho>0$

$$
f_{B_{\rho}\left(x_{0}\right)}\left|f(x, z)-f\left(x_{0}, z\right)\right| d x \leqslant C M^{\beta-1} \varepsilon+f_{B_{\rho}\left(x_{0}\right)}\left|f\left(x, z_{i}\right)-f\left(x_{0}, z_{i}\right)\right| d x
$$

and since $x_{0} \in \bigcap K_{i}$, we now have

$$
\limsup _{\rho \rightarrow 0} f_{B_{\rho}\left(x_{0}\right)}\left|f(x, z)-f\left(x_{0}, z\right)\right| d x \leqslant C M^{\beta-1} \varepsilon .
$$

The assertion follows by letting $\varepsilon \rightarrow 0$.

For every open set $A \subset \Omega$ and every $u \in W_{\text {loc }}^{1,1}\left(A ; \mathbb{R}^{d}\right)$, we set

$$
F(u, A):=\int_{A} f(x, \nabla u(x)) d x .
$$


Given an open set $A \subset \Omega$ and $u \in W_{\text {loc }}^{1,1}\left(A ; \mathbb{R}^{d}\right)$, the set of admissible sequences for $u$ in $A$ is defined by

$$
\mathcal{A}(u ; A):=\left\{\left\{u_{n}\right\} \subset W_{\mathrm{loc}}^{1, \beta}(A): u_{n} \rightarrow u \text { in } L^{1}\left(A ; \mathbb{R}^{d}\right)\right\},
$$

and we introduce the relaxed functional

$$
\mathcal{F}(u, A):=\inf \left\{\liminf _{n \rightarrow+\infty} F\left(u_{n}, A\right):\left\{u_{n}\right\} \in \mathcal{A}(u, A)\right\} .
$$

We define the set of good sequences for $u$ in $A$ to be

$$
\mathcal{G}(u, A):=\left\{\left\{u_{n}\right\} \in \mathcal{A}(u, A): \mathcal{F}(u, A)=\lim _{n \rightarrow+\infty} F\left(u_{n}, A\right)\right\} .
$$

It is clear that $\mathcal{G}(u, A)$ is nonempty. If $F(u, A)<+\infty$ then by (H3) we have that $\nabla u \in L^{\alpha}\left(A ; \mathbb{R}^{d \times N}\right)$, and so for almost all balls $B_{\rho} \subset A$ the trace of $u$ on $\partial B_{\rho}$ belongs to $W^{1, \alpha}\left(\partial B_{\rho} ; \mathbb{R}^{d}\right)$. In view of Lemma 2.1 , we may define for every such ball

$$
\mathbf{m}\left(u, B_{\rho}\right):=\inf \left\{F\left(v, B_{\rho}\right): v \in W^{1, \beta}\left(B_{\rho}\right), v-u \in W_{0}^{1, \alpha}\left(B_{\rho}\right)\right\},
$$

and we also set

$$
\mathcal{F}_{\mathrm{av}}(u, A):=\frac{1}{|A|} \mathcal{F}(u, A), \quad \mathbf{m}_{\mathrm{av}}\left(u, B_{\rho}\right)=\frac{1}{\left|B_{\rho}\right|} \mathbf{m}\left(u, B_{\rho}\right),
$$

where $B_{\rho}$ stands for a ball $B(x, \rho)$ of center $x \in \Omega$ and radius $\rho>0$.

Next we recall a semicontinuity result by Ioffe (see [19]).

THEOREM 2.4. - Let $g: \Omega \times \mathbb{R}^{d} \times \mathbb{R}^{d \times N} \rightarrow[0,+\infty)$ be a Carathéodory function such that $g(x, u, \cdot)$ is convex for every $u \in \mathbb{R}^{d}$ and for $\mathcal{L}^{N}$ a.e. $x \in \Omega$. Then the functional

$$
G(u):=\int_{\Omega} g(x, u, \nabla u) d x
$$

is lower semicontinuous on $W^{1,1}\left(\Omega ; \mathbb{R}^{d}\right)$ with respect to the weak convergence in $W^{1,1}\left(\Omega ; \mathbb{R}^{d}\right)$.

By Ioffe's Theorem, the functional $F$ is lower semicontinuous, thus

$$
F \leqslant \mathcal{F} \text {. }
$$

On the other hand, if $u \in W_{\text {loc }}^{1, \beta}\left(A ; \mathbb{R}^{d}\right) \cap W^{1, \alpha}\left(A ; \mathbb{R}^{d}\right)$ then the constant sequence $\{u\}$ is admissible and thus $\mathcal{F}(u, A) \leqslant F(u, A)$. This, together with the previous inequality, yields

$$
\mathcal{F}(u, A)=F(u, A) \quad \text { whenever } u \in W_{\text {loc }}^{1, \beta}\left(A ; \mathbb{R}^{d}\right) \cap W^{1, \alpha}\left(A ; \mathbb{R}^{d}\right) .
$$

Using a good sequence in $\mathcal{G}(u, A)$ to approach $\mathcal{F}(u, A)$, and in view of (2.5), it now follows that

$$
\mathcal{F}=\sup \left\{H: H \leqslant \bar{F}, H \text { is } L^{1} \text { lower semicontinuous }\right\}
$$


where

$$
\bar{F}(u, A):= \begin{cases}F(u, A) & \text { if } u \in W_{\mathrm{loc}}^{1, \beta}\left(A ; \mathbb{R}^{d}\right), \\ +\infty & \text { otherwise. }\end{cases}
$$

The following lemma will be instrumental to show that $\mathcal{F}(u, \cdot)$ is the trace in $\mathbb{A}(\Omega)$ of a positive Radon measure $\mu(u, \cdot)$, where $\mathbb{A}(\Omega)$ denotes the class of open subsets of $\Omega$.

LEMMA 2.5. - Let $\lambda: \mathbb{A}(\Omega) \rightarrow[0,+\infty)$ and $\mu$ be such that

(i) $\mu$ is a finite Radon measure on $\Omega$;

(ii) $\lambda(\Omega) \geqslant \mu(\Omega)$;

(iii) $\lambda(A) \leqslant \mu(\bar{A})$ for all $A \in \mathbb{A}(\Omega)$;

(iv) (subadditivity) $\lambda(A) \leqslant \lambda(A \backslash \bar{C})+\lambda(B)$ for all $A, B, C \in \mathbb{A}(\Omega)$ such that $C \Subset$ $B \subset A$;

(v) (inner regularity) for all $A \in \mathbb{A}(\Omega), \varepsilon>0$, there exists $C \in \mathbb{A}(\Omega)$ such that $C \Subset A$ and $\lambda(A \backslash \bar{C})<\varepsilon$.

Then $\lambda=\mu$ on $\mathbb{A}(\Omega)$.

Proof. - We start by showing that

$$
\lambda(A) \leqslant \mu(A) \text { for all } A \in \mathbb{A}(\Omega) .
$$

Fix $A \in \mathbb{A}(\Omega), \varepsilon>0$. By (iv) and (v) we may find open sets $C \Subset B \Subset A$ such that $\lambda(A \backslash \bar{C})<\varepsilon$ and

$$
\lambda(A) \leqslant \lambda(A \backslash \bar{C})+\lambda(B) \leqslant \varepsilon+\mu(\bar{B}) \leqslant \varepsilon+\mu(A),
$$

where we used (iii). Clearly (2.6) now follows by letting $\varepsilon \rightarrow 0^{+}$.

In order to prove the converse inequality, since $\mu$ is inner regular for $\varepsilon>0$ we may find an open set $A^{\prime} \Subset A$ such that $\mu(A) \leqslant \varepsilon+\mu\left(\overline{A^{\prime}}\right)$, and by virtue of (ii), (2.6), and (iv),

$$
\mu(A) \leqslant \varepsilon+\mu\left(\overline{A^{\prime}}\right)=\varepsilon+\mu(\Omega)-\mu\left(\Omega \backslash \overline{A^{\prime}}\right) \leqslant \varepsilon+\lambda(\Omega)-\lambda\left(\Omega \backslash \overline{A^{\prime}}\right) \leqslant \varepsilon+\lambda(A) .
$$

By the arbitrariness of $\varepsilon>0$, we now conclude that $\mu(A) \leqslant \lambda(A)$.

Remark 2.6. - If (i) and (iii) in the statement of Lemma 2.5 hold, then the inner regularity property (v) is satisfied provided we can show that there exists $C>0$ such that if $U \in \mathbb{A}(\Omega), U=\bigcup_{i=1}^{\infty} U_{i}, U_{i} \in \mathbb{A}(\Omega), U_{i} \Subset U_{i+1} \Subset U$, then

$$
\lambda\left(U \backslash \overline{U_{1}}\right) \leqslant C \sum_{i=1}^{\infty} \lambda\left(U_{i+3} \backslash \overline{U_{i}}\right) .
$$

Indeed, if $U \in \mathbb{A}(\Omega)$, take $U_{i}:=\{x \in U: \operatorname{dist}(x, \partial U)>1 / i\}$, and choose $k$ large enough so that

$$
\mathbf{m}\left(u, U \backslash U_{k}\right)<\frac{\varepsilon}{4 C} .
$$

We have 


$$
\begin{aligned}
\lambda\left(U \backslash \overline{U_{k}}\right) & \leqslant C \sum_{i=k}^{\infty} \lambda\left(U_{i+3} \backslash \overline{U_{i}}\right) \leqslant C \sum_{i=k}^{\infty} \mu\left(\overline{U_{i+3} \backslash \overline{U_{i}}}\right) \\
& \leqslant C \sum_{i=k}^{\infty} \mu\left(U_{i+4} \backslash U_{i}\right) \leqslant 4 C \mu\left(U \backslash U_{k}\right)<\varepsilon .
\end{aligned}
$$

\section{A representation theorem}

In this section we prove Theorem 1.1. We start by showing that $\mathcal{F}(u, \cdot)$ is the trace in $\mathbb{A}(\Omega)$ of a Radon measure $\mu(u, \cdot)$. For every $A \in \mathbb{A}(\Omega)$ set

$$
\lambda(A):=\mathcal{F}(u, A) .
$$

Consider a sequence $\left\{u_{n}\right\} \in \mathcal{G}(u, A)$ such that

$$
\mathcal{F}(u, \Omega)=\lim _{n \rightarrow+\infty} F\left(u_{n}, \Omega\right)=\lim _{n \rightarrow+\infty} \int_{\Omega} f\left(x, \nabla u_{n}\right) d x,
$$

and define the Radon measures on $\mathbb{R}^{N}$

$$
\lambda_{n}:=f\left(x, \nabla u_{n}\right) \mathcal{L}^{N}\llcorner\Omega .
$$

Since these measures are equibounded, and up to the extraction of a subsequence, we have

$$
\lambda_{n} \stackrel{*}{\rightarrow} \mu(u, \cdot) \quad \text { in the sense of measures }
$$

for some finite Radon measure $\mu(u, \cdot)$, i.e.,

$$
\int_{\Omega} \varphi d \lambda_{n} \rightarrow \int_{\Omega} \varphi d \mu(u, \cdot) \quad \text { for all } \varphi \in C_{c}(\Omega) .
$$

Note that

$$
\mu(u, \Omega) \leqslant \liminf _{n \rightarrow+\infty} \lambda_{n}(\Omega)=\lim _{n \rightarrow+\infty} \int_{\Omega} f\left(x, \nabla u_{n}\right) d x=\lambda(\Omega),
$$

and if $A \in \mathbb{A}(\Omega)$ then

$$
\lambda(A) \leqslant \liminf _{n \rightarrow+\infty} \int_{A} f\left(x, \nabla u_{n}\right) d x=\liminf _{n \rightarrow+\infty} \lambda_{n}(A) \leqslant \mu(u, \bar{A}) .
$$

Therefore, by virtue of Lemma 2.5 we have

$$
\lambda=\mu(u, \cdot) \quad \text { on } \mathbb{A}(\Omega)
$$

provided $\lambda$ satisfies the subadditivity and inner regularity properties (iv) and (v). These depend mostly on the growth condition (H3) and on (2.1), and were asserted in [15] 
(see Lemma 2.4) by means of an uplifting operator $\mathcal{P}$ more refined and complex than the operator $E_{A}$ introduced in Lemma 2.1. Below we give an alternative proof which relies heavily on the convexity assumption (H2), and it bypasses the need to exploit the operator $\mathcal{P}$.

Proposition 3.1. - If $A, B, C \in \mathbb{A}(\Omega), C \Subset B \subset A$, then

$$
\mathcal{F}(u, A) \leqslant \mathcal{F}(u, B)+\mathcal{F}(u, A \backslash \bar{C}) .
$$

Proof. - Fix $\left\{v_{n}\right\} \in \mathcal{G}(u, B)$ and $\left\{w_{n}\right\} \in \mathcal{G}(u, A \backslash \bar{C})$, let $\varphi \in C_{c}^{\infty}(B)$ be such that $0 \leqslant \varphi \leqslant 1, \varphi=1$ in a neighborhood of $C$, and set

$$
u_{n}:=\varphi v_{n}+(1-\varphi) w_{n},
$$

where $\varphi v_{n}$ is taken to be zero there where $\varphi=0$, even though $v_{n}$ may be not defined on that set, and analogously $(1-\varphi) w_{n}$ is to be equal to zero on $\{\varphi=1\}$. Clearly $\left\{u_{n}\right\} \in \mathcal{A}(u, A)$, and for all $0<t<1$ also $\left\{t u_{n}\right\} \in \mathcal{A}(t u, A)$, thus

$$
\mathcal{F}(t u, A) \leqslant \liminf _{n \rightarrow+\infty} F\left(t u_{n}, A\right) .
$$

By the convexity of $f$, and since $0<t<1,0 \leqslant \varphi \leqslant 1$, we may find some positive constant $M$ such that

$$
\begin{aligned}
F\left(t u_{n}, A\right)= & \int_{A} f\left(x, t\left[\varphi \nabla v_{n}+(1-\varphi) \nabla w_{n}\right]+(1-t) \frac{v_{n}-w_{n}}{1-t} t \nabla \varphi\right) d x \\
\leqslant & t \int_{A} f\left(x, \varphi \nabla v_{n}+(1-\varphi) \nabla w_{n}\right) d x \\
& +(1-t) \int_{A} M\left(1+\left|\frac{v_{n}-w_{n}}{1-t} t \nabla \varphi\right|^{\beta}\right) d x \\
\leqslant & \int_{A}\left[\varphi(x) f\left(x, \nabla v_{n}\right)+(1-\varphi(x)) f\left(x, \nabla w_{n}\right)\right] d x+M(1-t)|A| \\
& +M \frac{\|\nabla \varphi\|_{L^{\infty}}^{\beta}}{(1-t)^{\beta-1}} \int_{B \backslash \bar{C}}\left|v_{n}-w_{n}\right|^{\beta} d x \\
\leqslant & F\left(v_{n}, B\right)+F\left(w_{n}, A \backslash \bar{C}\right)+M(1-t)|A| \\
& +M \frac{\|\nabla \varphi\|_{L^{\infty}}^{\beta}\left\|v_{n}-w_{n}\right\|_{L^{\beta}(B \backslash \bar{C})}^{\beta},}{(1-t)^{\beta-1}}
\end{aligned}
$$

where we have used the growth condition in (H3). Also, by the coercivity assumption in (H3), we deduce that $\left\{\left\|\nabla v_{n}\right\|_{L^{\alpha}(B \backslash \bar{C})}+\left\|\nabla w_{n}\right\|_{L^{\alpha}(B \backslash \bar{C})}\right\}$ is bounded, and since $v_{n}-w_{n} \rightarrow$ $u-u=0$ in $L^{1}\left(B \backslash \bar{C} ; \mathbb{R}^{d}\right)$, we have that $v_{n}-w_{n} \rightarrow 0$ weakly in $W^{1, \alpha}\left(B \backslash \bar{C} ; \mathbb{R}^{d}\right)$, and therefore strongly in $L^{\beta}\left(B \backslash \bar{C} ; \mathbb{R}^{d}\right)$ by (2.1) and Rellich Theorem. This, together with (3.3), yields

$$
\mathcal{F}(t u, A) \leqslant \mathcal{F}(u, B)+\mathcal{F}(u, A \backslash \bar{C})+C(1-t)|A|,
$$


and the result now follows by letting $t \nearrow 1$ and using the $L^{1}$ lower semicontinuity property of $\mathcal{F}(\cdot, A)$.

Finally, and in light of Remark 2.6, in order to assert inner regularity for $\lambda$ it suffices to establish (2.7).

Proposition 3.2. - If $U \in \mathbb{A}(\Omega), U=\bigcup_{i=1}^{\infty} U_{i}, U_{i} \in \mathbb{A}(\Omega), U_{i} \Subset U_{i+1} \Subset U$, then

$$
\mathcal{F}\left(u, U \backslash \overline{U_{1}}\right) \leqslant 2 \sum_{i=1}^{\infty} \mathcal{F}\left(u, U_{i+3} \backslash \overline{U_{i}}\right) .
$$

Proof. - Fix $U \in \mathbb{A}(\Omega)$ and suppose that $U=\bigcup_{i=1}^{\infty} U_{i}, U_{i} \in \mathbb{A}(\Omega), U_{i} \Subset U_{i+1} \Subset U$. Choose $\left\{u_{n}^{(i)}\right\} \in \mathcal{G}\left(u, U_{i+2} \backslash \overline{U_{i}}\right)$ and $\left\{u_{n}^{\infty}\right\} \in \mathcal{G}(u, U)$, so that, recalling that $\lambda=\mathcal{F}(u, \cdot)$,

$$
\lambda\left(U_{i+2} \backslash \overline{U_{i}}\right)=\lim _{n \rightarrow+\infty} \int_{U_{i+2} \backslash \overline{U_{i}}} f\left(x, \nabla u_{n}^{(i)}\right) d x, \quad \lambda(U)=\lim _{n \rightarrow+\infty} \int_{U} f\left(x, \nabla u_{n}^{\infty}(x)\right) d x .
$$

Let $\varphi_{i}$ be a smooth cut-off function such that $\varphi_{i}=1$ in $U_{i}, \varphi_{i}=0$ outside $U_{i+1}$, and $\left\{0<\varphi_{i}<1\right\} \Subset U_{i+1} \backslash \overline{U_{i}}$.

Up to the extraction of a subsequence, we may assume that $f\left(\cdot, \nabla u_{n}^{\infty}\right)\llcorner U \stackrel{*}{\rightarrow} \eta$, where $\eta$ is a finite Radon measure on $U$. Fix $\varepsilon>0$ and choose $M \in \mathbb{N}$ large enough so that $\eta\left(U \backslash \overline{U_{M}}\right)<\varepsilon$. Define

$$
\begin{aligned}
\bar{u}_{n}:= & \chi_{U_{2} \backslash \overline{U_{1}}} u_{n}^{(1)}+\sum_{i=2}^{M} \chi_{U_{i+1} \backslash U_{i}}\left[\varphi_{i} u_{n}^{(i-1)}+\left(1-\varphi_{i}\right) u_{n}^{(i)}\right] \\
& +\chi_{U \backslash U_{M+1}}\left[\varphi_{M+1} u_{n}^{(M)}+\left(1-\varphi_{M+1}\right) u_{n}^{\infty}\right] .
\end{aligned}
$$

Clearly $\bar{u}_{n} \in \mathcal{A}\left(u, U \backslash \overline{U_{1}}\right)$, and we have

$$
\begin{aligned}
\lambda\left(U \backslash \overline{U_{1}}\right) \leqslant & \liminf _{t \rightarrow 1} \liminf _{n \rightarrow+\infty} \int_{U \backslash \overline{U_{1}}} f\left(x, t \nabla \bar{u}_{n}\right) d x \\
\leqslant & \limsup _{t \rightarrow 1} \limsup _{n \rightarrow+\infty} t \int_{U_{2} \backslash \overline{U_{1}}} f\left(x, \nabla u_{n}^{(1)}\right) d x+\limsup _{t \rightarrow 1}(1-t) \int_{U_{2} \backslash \overline{U_{1}}} f(x, 0) d x \\
& +\limsup _{t \rightarrow 1} \limsup _{n \rightarrow+\infty} \sum_{i=2}^{M} \int_{U_{i+1} \backslash U_{i}} f\left(x, t \nabla \bar{u}_{n}\right) d x \\
& +\limsup _{t \rightarrow 1} \limsup _{n \rightarrow+\infty} \int_{U \backslash U_{M+1}} f\left(x, t \nabla \bar{u}_{n}\right) .
\end{aligned}
$$

Now

$$
\limsup _{t \rightarrow 1} \limsup _{n \rightarrow+\infty} t \int_{U_{2} \backslash \overline{U_{1}}} f\left(x, \nabla u_{n}^{(1)}\right) d x+\limsup _{t \rightarrow 1}(1-t) \int_{U_{2} \backslash \overline{U_{1}}} f(x, 0) d x \leqslant \lambda\left(U_{3} \backslash \overline{U_{1}}\right),
$$


and

$$
\begin{aligned}
\underset{t \rightarrow 1}{\limsup } \limsup _{n \rightarrow+\infty} \sum_{i=2}^{M} \int_{U_{i+1} \backslash U_{i}} f\left(x, t \nabla \bar{u}_{n}\right) d x \\
=\limsup _{t \rightarrow 1} \limsup _{n \rightarrow+\infty} \sum_{i=2}^{M} \int_{U_{i+1} \backslash U_{i}} f\left(x, t\left[\varphi_{i} \nabla u_{n}^{(i-1)}+\left(1-\varphi_{i}\right) \nabla u_{n}^{(i)}\right]\right. \\
\left.\quad+(1-t) \frac{u_{n}^{(i-1)}-u_{n}^{(i)}}{1-t} t \nabla \varphi_{i}\right) d x \\
\leqslant \\
\quad \limsup _{t \rightarrow 1} \limsup _{n \rightarrow+\infty} t \sum_{i=2}^{M}\left\{\int_{U_{i+1} \backslash \overline{U_{i-1}}} \varphi_{i} f\left(x, \nabla u_{n}^{(i-1)}\right) d x\right. \\
\left.\quad+\int_{U_{i+2} \backslash \overline{U_{i}}}\left(1-\varphi_{i}\right) f\left(x, \nabla u_{n}^{(i)}\right) d x\right\} \\
\quad+\limsup _{t \rightarrow 1} \limsup _{n \rightarrow+\infty}(1-t) \sum_{i=2}^{M} \int{ }_{U_{i+1} \backslash \overline{U_{i}}} C\left(1+\frac{\left|u_{n}^{(i-1)}-u_{n}^{(i)}\right|^{\beta}}{(1-t)^{\beta}} t^{\beta}\left|\nabla \varphi_{i}\right|^{\beta}\right) d x \\
\leqslant \sum_{i=2}^{M}\left[\lambda\left(U_{i+1} \backslash \overline{U_{i-1}}\right)+\lambda\left(U_{i+2} \backslash \overline{U_{i}}\right)\right],
\end{aligned}
$$

where we have used the fact that $u_{n}^{(i-1)}-u_{n}^{(i)} \rightarrow 0$ in $L^{\beta}\left(U_{i+1} \backslash \overline{U_{i}}\right)$.

In a similar way, we can show that

$$
\begin{aligned}
& \limsup _{t \rightarrow 1} \limsup _{n \rightarrow+\infty} \int_{U \backslash U_{M+1}} f\left(x, t \nabla \bar{u}_{n}\right) \\
& \quad \leqslant \limsup _{n \rightarrow+\infty} \int_{U_{M+2} \backslash U_{M+1}} f\left(x, \nabla u_{n}^{(M)}\right)+\limsup _{n \rightarrow+\infty} \int_{U \backslash U_{M+1}} f\left(x, \nabla u_{n}^{\infty}\right) d x \\
& \quad \leqslant \lambda\left(U_{M+3} \backslash \overline{U_{M}}\right)+\eta\left(U \backslash \overline{U_{M}}\right) \\
& \quad \leqslant \lambda\left(U_{M+3} \backslash \overline{U_{M}}\right)+\varepsilon .
\end{aligned}
$$

In view of (3.4), (3.5), (3.6), and (3.7), we obtain

$$
\lambda\left(U \backslash \overline{U_{1}}\right) \leqslant 2 \sum_{i=1}^{\infty} \lambda\left(U_{i+3} \backslash \overline{U_{i}}\right)+\varepsilon .
$$

Letting $\varepsilon \rightarrow 0$ yields property (2.7).

Proposition 3.3. - If $(\mathrm{H} 1)-(\mathrm{H} 3)$ hold and if $\mathcal{F}(u, \Omega)<+\infty$ then there exists $a$ finite Radon measure $\mu(u, \cdot)$ on $\Omega$ such that $\mathcal{F}(u, \cdot)$ is the trace of $\mu(u, \cdot)$ on $\mathbb{A}(\Omega)$.

Proof. - The proof follows immediately from Lemma 2.5, Remark 2.6, (3.1), (3.2), Propositions 3.1 and 3.2. 
In the sequel we will often write $\mu(u, \cdot)$ to designate the measure whose trace on the open sets of $\Omega$ is $\mathcal{F}(u, \cdot)$, and we will denote by $\mu^{s}(u, \cdot)$ its singular part with respect to the Lebesgue measure $\mathcal{L}^{N}\llcorner\Omega$.

LEMMA 3.4. - If $(\mathrm{H} 1)-(\mathrm{H} 3)$ hold and if $\mathcal{F}(u, \Omega)<+\infty$ then there exists a sequence $\left\{u_{n}\right\}$ with the following properties:

(a) $u_{n} \in W_{\text {loc }}^{1, \beta}(\Omega)$;

(b) $u_{n} \rightarrow u$ in $W^{1, \alpha}(\Omega)$;

(c) $\mathcal{F}(u, \Omega)=\lim _{n \rightarrow+\infty} F\left(u_{n}, \Omega\right)$;

(d) $\mathcal{F}(u, A)=\lim _{n \rightarrow+\infty} F\left(u_{n}, A\right)$ for all open sets $A \subset \Omega$ such that $\mu(u, \partial A)=0$;

(e) for all $x_{0} \in \Omega$ and for almost all $0<t<R:=\operatorname{dist}\left(x_{0}, \partial \Omega\right)$, there exists a subsequence $\left\{u_{k_{n}}\right\}$ such that $\mathcal{F}\left(u, B_{t}\left(x_{0}\right)\right)=\lim _{n \rightarrow+\infty} F\left(u_{k_{n}}, B_{t}\left(x_{0}\right)\right)$ and $u_{k_{n}} \rightarrow u$ in $W^{1, \alpha}\left(\partial B_{t}\left(x_{0}\right)\right)$.

Proof. - By the definition of $\mathcal{F}$ there exists a sequence $\left\{u_{n}\right\}$ satisfying properties (a), (b), (c). If $A \in \mathbb{A}(\Omega)$ is such that $\mu(u, \partial A)=0$ then

$$
\begin{aligned}
\limsup _{n \rightarrow+\infty} F\left(u_{n}, A\right) & =\lim _{n \rightarrow+\infty} F\left(u_{n}, \Omega\right)-\liminf _{n \rightarrow+\infty} F\left(u_{n}, \Omega \backslash \bar{A}\right) \\
& \leqslant \mathcal{F}(u, \Omega)-\mathcal{F}(u, \Omega \backslash \bar{A}) \\
& =\mathcal{F}(u, A) \\
& \leqslant \liminf _{n \rightarrow+\infty} F\left(u_{n}, A\right)
\end{aligned}
$$

and property (d) follows. To prove (e), fix $x_{0} \in \Omega$ and remark that due to the finiteness of the measure $\mu(u, \cdot)$, property (d) holds for $B_{t}\left(x_{0}\right)$ for $\mathcal{L}^{1}$ a.e. $t \in(0, R)$. With

$$
M:=\sup _{n} \int_{B_{R}\left(x_{0}\right)}\left|\nabla u_{n}\right|^{\alpha} d x<+\infty,
$$

define for $K>0$

$$
E_{K, n}=\bigcap_{m \geqslant n}\left\{t \in(0, R): \mu_{s}\left(u, \partial B_{t}\left(x_{0}\right)\right)=0, \int_{\partial B_{t}\left(x_{0}\right)}\left|\nabla u_{m}\right|^{\alpha} d H^{N-1} \geqslant K\right\},
$$

where $\mu_{s}(u, \cdot)$ is the singular part of $\mu(u, \cdot)$ with respect to $\mathcal{L}^{N}\left\llcorner\Omega\right.$. Clearly $\left|E_{K, n}\right| \leqslant$ $M / K$, and since $E_{K, n+1} \supset E_{K, n}$, we still have $\left|E_{K}\right| \leqslant M / K$ where $E_{K}:=\bigcup_{n} E_{K, n}$. As a consequence, if $E:=\bigcap_{K} E_{K}$ then $|E|=0$, and if $t \notin E$ then

$$
\liminf _{n \rightarrow+\infty} \int_{\partial B_{t}\left(x_{0}\right)}\left|\nabla u_{n}\right|^{\alpha} d H^{N-1}<+\infty
$$

which, together with (d), asserts the existence of a subsequence fulfilling (e).

DEFINITION 3.5. - If $\mathcal{F}(u, \Omega)<+\infty$ then we say that $B_{\rho}\left(x_{0}\right)$ is an uplift ball for $u$ if:

(i) $\mu\left(u, \partial B_{\rho}\left(x_{0}\right)\right)=0($ where $\mu(u, \cdot)$ is the measure given by Proposition 3.3). 
(ii) There exists a sequence $\left\{u_{n}\right\}$ satisfying properties (a), (b), (c) of Lemma 3.4 and $u_{n} \rightarrow u$ weakly in $W^{1, \alpha}\left(\partial B_{\rho}\left(x_{0}\right)\right)$.

We denote by $\mathcal{R}\left(u, x_{0}\right)$ the set of all radii $\rho>0$ such that $B_{\rho}\left(x_{0}\right)$ is an uplift ball.

In the sequel, we shall often deal with limits involving the function $\mathbf{m}\left(u, B_{\rho}(x)\right)$ as $\rho \rightarrow 0$. Since $\mathbf{m}$ is defined only for $\rho \in \mathcal{R}(u, x)$, i.e. for almost all $\rho$, and is not defined for the remaining values of $\rho$, as it is customary for a given function $f$ when we write $\lim _{\rho \rightarrow 0} f(\rho)$ it should be understood that $\rho$ is taken only among those points at which the function $f$ is defined.

Remark 3.6. - By Lemma 3.4, if $\mathcal{F}(u, \Omega)<+\infty$ then for all $x_{0} \in \Omega$ almost all balls centered at $x_{0}$ are uplift balls for $u$. Also, by means of a diagonal argument we may assume that the sequence $\left\{u_{n}\right\}$ satisfying (ii) in Definition 3.5 is the same for a countable set of uplift balls.

LEMMA 3.7. - Assume that $\mathcal{F}(u, \Omega)<+\infty$ and let $B$ be an uplift ball for $u$. Let $v \in W^{1, \beta}\left(B ; \mathbb{R}^{d}\right)$ satisfy $v=u$ on $\partial B$, set

$$
\bar{u}(x):= \begin{cases}v(x) & \text { if } x \in B \\ u(x) & \text { otherwise. }\end{cases}
$$

Then

$$
\mu(\bar{u}, \partial B)=0 .
$$

Proof. - Let $\left\{u_{n}\right\}$ be a sequence as in Definition 3.5(ii), and let $B^{\prime} \Subset B \Subset B^{\prime \prime}$ be uplift balls concentric with $B$, so close together that if we denote by $A$ the annulus $B^{\prime \prime} \backslash \overline{B^{\prime}}$, and using the fact that $\mathcal{F}(u, \partial B)=0$, then

$$
\mathcal{F}(u, A)<\varepsilon, \quad F(v, A \cap B)<\varepsilon .
$$

Since the trace on $\partial B$ of $u_{n}-u$ converges to zero weakly on $W^{1, \alpha}\left(\partial B ; \mathbb{R}^{d}\right)$, by Lemma 2.1 if we take $v_{n}:=\mathrm{E}_{B}\left(u_{n}-u\right)$ then

$$
v_{n} \in W^{1, \beta}(B), \quad v_{n}=u_{n}-u \quad \text { on } \partial B, \quad v_{n} \rightarrow 0 \quad \text { in } W^{1, \beta}\left(B ; \mathbb{R}^{d}\right) .
$$

We define in $A$

$$
\bar{u}_{n}(x):= \begin{cases}u_{n}(x) & \text { if } x \in A \backslash B, \\ v_{n}(x)+v(x) & \text { if } x \in A \cap B\end{cases}
$$

Clearly $\bar{u}_{n} \in W^{1, \beta}\left(A ; \mathbb{R}^{d}\right)$, and

$$
F\left(\bar{u}_{n}, A\right)=F\left(u_{n}, A \backslash B\right)+F\left(\bar{u}_{n}, A \cap B\right) \leqslant F\left(u_{n}, A\right)+F\left(\bar{u}_{n}, A \cap B\right) .
$$

Since $\bar{u}_{n} \rightarrow \bar{u}$ in $L^{1}\left(A ; \mathbb{R}^{d}\right)$ and $\bar{u}_{n} \rightarrow v$ in $W^{1, \beta}\left(A \cap B ; \mathbb{R}^{d}\right)$, by the $W^{1, \beta}$-continuity of $F$ and the property (d) satisfied by $\left\{u_{n}\right\}$, in view of the fact that $\mu(u, \partial A)=0$, we deduce that

$$
\mathcal{F}(\bar{u}, A) \leqslant \liminf _{n \rightarrow+\infty} F\left(\bar{u}_{n}, A\right)
$$




$$
\begin{aligned}
& \leqslant \limsup _{n \rightarrow+\infty} F\left(\bar{u}_{n}, A \backslash B\right)+\limsup _{n \rightarrow+\infty} F\left(\bar{u}_{n}, A \cap B\right) \\
& \leqslant \lim _{n \rightarrow+\infty} F\left(u_{n}, A\right)+F(v, A \cap B) \\
& =\mathcal{F}(u, A)+F(v, A \cap B)<2 \varepsilon
\end{aligned}
$$

and the assertion follows.

Proposition 3.8. - If $\mathcal{F}(u, \Omega)<+\infty$ then for $\mathcal{L}^{N}$-a.e. $x_{0} \in \Omega$

$$
\liminf _{\rho \rightarrow 0} \mathbf{m}_{\mathrm{av}}\left(u, B_{\rho}\left(x_{0}\right)\right) \leqslant f\left(x_{0}, \nabla u\left(x_{0}\right)\right) .
$$

Proof. - We use the notation introduced in Remark 2.3. We recall that since $u \in$ $W_{\text {loc }}^{1, \alpha}\left(\Omega ; \mathbb{R}^{d}\right)$, for $\mathcal{L}^{N}$-a.e. point $x_{0}$ and every sequence $\rho_{j} \searrow 0$ we have (see [13], Theorem 2, p. 230)

$$
\underset{B_{\rho_{j}}\left(x_{0}\right)}{f}\left[\left|\nabla u(x)-\nabla u\left(x_{0}\right)\right|^{\alpha}+\frac{1}{\rho_{j}^{\alpha}}\left|u(x)-u\left(x_{0}\right)-\nabla u\left(x_{0}\right)\left(x-x_{0}\right)\right|^{\alpha}\right] d x \rightarrow 0 .
$$

Let $x_{0} \in \mathbb{K}(f)$ be any such point, which in addition is a Lebesgue point for $u$ and $\nabla u$, and let $\rho_{j} \searrow 0$ be any sequence such that $B_{\rho_{1}}\left(x_{0}\right) \subset \Omega$. On $B:=B_{1}(0)$ we define

$$
u_{j}(y):=\frac{1}{\rho_{j}}\left[u\left(x_{0}+\rho_{j} y\right)-u\left(x_{0}\right)\right], \quad u_{0}(y):=\nabla u\left(x_{0}\right) y .
$$

Then

$$
f_{B}\left[\left|\nabla u_{j}-\nabla u_{0}\right|^{\alpha}+\left|u_{j}-u_{0}\right|^{\alpha}\right] d y \rightarrow 0,
$$

i.e.,

$$
\int_{0}^{1} d t \int_{\partial B_{t}}\left[\left|\nabla u\left(x_{0}+\rho_{j} y\right)-\nabla u\left(x_{0}\right)\right|^{\alpha}+\left|u_{j}(y)-\nabla u\left(x_{0}\right) y\right|^{\alpha}\right] d H^{N-1} \rightarrow 0,
$$

where $B_{t}:=B_{t}(0)$, and so, up to a subsequence,

$$
\int_{\partial B_{t}}\left[\left|\nabla u\left(x_{0}+\rho_{j} y\right)-\nabla u\left(x_{0}\right)\right|^{\alpha}+\left|u_{j}(y)-\nabla u\left(x_{0}\right) y\right|^{\alpha}\right] d H^{N-1} \rightarrow 0
$$

for $\mathcal{L}^{1}$ a.e. $t \in(0,1)$. As for almost all $t$

$$
\mu\left(u, \partial B_{t \rho_{j}}\right)=0 \quad \text { and } \quad u_{j} \in W^{1, \alpha}\left(\partial B_{t} ; \mathbb{R}^{d}\right) \quad \text { for all } j,
$$

we may find $\bar{t} \in(0,1)$ satisfying (3.9), (3.10), and we relabel as $\left\{\rho_{j}\right\}$ the sequence $\left\{\bar{t} \rho_{j}\right\}$. We then have that the sequence defined in (3.8) with the new $\rho_{j}$ satisfies 


$$
\begin{aligned}
& \int_{\partial B}\left[\left|\nabla u\left(x_{0}+\rho_{j} y\right)-\nabla u\left(x_{0}\right)\right|^{\alpha}+\left|u_{j}(y)-\nabla u\left(x_{0}\right) y\right|^{\alpha}\right] d H^{N-1} \rightarrow 0, \\
& \mu\left(u, \partial B_{\rho_{j}}\right)=0, \quad u_{j} \in W^{1, \alpha}\left(\partial B ; \mathbb{R}^{d}\right) \quad \text { for all } j
\end{aligned}
$$

and $u_{j}$ still converges to $u_{0}$ in $W^{1, \alpha}\left(B ; \mathbb{R}^{d}\right)$. Setting $v_{j}:=\mathrm{E}_{B} u_{j}$, and as $\mathrm{E}_{B} u_{0}=u_{0}$ (see Remark 2.2), from Lemma 2.1 and (3.11) we deduce that

$$
v_{j} \rightarrow u_{0} \quad \text { in } W^{1, \beta}\left(B ; \mathbb{R}^{d}\right)
$$

and

$$
\begin{aligned}
\mathbf{m}_{\mathrm{av}}\left(u, B_{\rho_{j}}\left(x_{0}\right)\right) & \leqslant \int_{B_{\rho_{j}}} f\left(x, \nabla\left(\rho_{j} v_{j}\left(\frac{x-x_{0}}{\rho_{j}}\right)+u\left(x_{0}\right)\right)\right) d y \\
& =f_{B} f\left(x_{0}+\rho_{j} y, \nabla v_{j}(y)\right) d y .
\end{aligned}
$$

Since $f$ is $\beta$-locally Lipschitz (see (2.2)), we obtaind

$$
\begin{aligned}
& \int_{B}\left|f\left(x_{0}+\rho_{j} y, \nabla v_{j}(y)\right)-f\left(x_{0}+\rho_{j} y, \nabla u\left(x_{0}\right)\right)\right| d y \\
& \quad \leqslant C \int_{B}\left(1+\left|\nabla v_{j}(y)\right|^{\beta-1}+\left|\nabla u\left(x_{0}\right)\right|^{\beta-1}\right)\left|\nabla v_{j}(y)-\nabla u\left(x_{0}\right)\right| d y \\
& \quad \leqslant C\left\|\nabla v_{j}-\nabla u_{0}\right\|_{L^{\beta}(B)}
\end{aligned}
$$

which vanishes as $j \rightarrow+\infty$. Therefore

$$
\begin{aligned}
\liminf _{\rho \rightarrow 0} \mathbf{m}_{\mathrm{av}}\left(u, B_{\rho}\left(x_{0}\right)\right) & \leqslant \limsup _{j \rightarrow+\infty} \mathbf{m}_{\mathrm{av}}\left(u, B_{\rho_{j}}\left(x_{0}\right)\right) \\
& \leqslant \limsup _{j \rightarrow+\infty} f_{B} f\left(x_{0}+\rho_{j} y, \nabla u\left(x_{0}\right)\right) d y \\
& =\limsup _{j \rightarrow+\infty} f f\left(x, \nabla u\left(x_{0}\right)\right) d x .
\end{aligned}
$$

By (3.12) and the definition of the set $\mathbb{K}(f)$ (see Remark 2.3), we conclude that

$$
\liminf _{\rho \rightarrow 0} \mathbf{m}_{\mathrm{av}}\left(u, B_{\rho}\left(x_{0}\right)\right) \leqslant f\left(x_{0}, \nabla u\left(x_{0}\right)\right) .
$$

Remark 3.9. - Recall that Ioffe's Theorem (Theorem 2.4) ensures that $F \leqslant \mathcal{F}$. In particular, if $\mathcal{F}(u, \Omega)<+\infty$ then $f(\cdot, \nabla u) \in L^{1}(\Omega)$. Moreover, if the point $x_{0}$ in the previous proof is also a Lebesgue point for $f(\cdot, \nabla u)$, which holds $\mathcal{L}^{N}$-a.e. in $\Omega$, then the 
sequences $\left\{\rho_{j}\right\}$ and $\left\{u_{j}\right\}$ we found above satisfy

$$
\left\{\begin{array}{l}
\mu_{s}\left(\partial B_{\rho_{j}}\right)=0, \\
u \in W^{1, \alpha}\left(\partial B_{\rho_{j}}\right), \\
u_{j} \rightarrow u_{0} \text { in } W^{1, \alpha}\left(B ; \mathbb{R}^{d}\right) \text { and in } W^{1, \alpha}\left(\partial B ; \mathbb{R}^{d}\right), \\
\limsup _{j \rightarrow+\infty} \mathbf{m}_{\mathrm{av}}\left(u, B_{\rho_{j}}\left(x_{0}\right)\right) \leqslant \lim _{j \rightarrow+\infty} f_{B_{\rho_{j}}\left(x_{0}\right)} f(x, \nabla u(x)) d x,
\end{array}\right.
$$

and from the last inequality we deduce, in particular, that for all $t>0$ there exists $\bar{j}$ such that for all $j \geqslant \bar{j}$

$$
\mathbf{m}\left(u, B_{\rho_{j}}\left(x_{0}\right)\right) \leqslant \int_{B_{\rho_{j}}\left(x_{0}\right)} f(x, \nabla u(x)) d x+t\left|B_{\rho_{j}}\left(x_{0}\right)\right| .
$$

Proposition 3.10. - For $\mathcal{L}^{N}$-a.e. $x_{0} \in \Omega$

$$
\liminf _{\rho \rightarrow 0} \mathcal{F}_{\text {av }}\left(u, B_{\rho}\left(x_{0}\right)\right) \leqslant f\left(x_{0}, \nabla u\left(x_{0}\right)\right) .
$$

Proof. - Fix an open set $\Omega^{\prime} \subset \Omega$ and define for every $t \in(0,1)$

$$
E_{t}:=\left\{x \in \Omega^{\prime}: \exists \rho_{j} \searrow 0 \text { such that } \mathbf{m}\left(u, B_{\rho_{j}}(x)\right) \leqslant \int_{B_{\rho_{j}}(x)} f(y, \nabla u(y)) d y+t\left|B_{\rho_{j}}(x)\right|\right\} .
$$

By Remark 3.9 we have that $\left|\Omega^{\prime} \backslash E_{t}\right|=0$, thus, in particular, $E_{t}$ is $\mathcal{L}^{N}$-measurable. Fix $\varepsilon>0$ and choose an open set $\omega$ and a compact set $K$ such that

$$
K \subset E_{t} \subset \omega \subset \Omega^{\prime}
$$

with

$$
\mu_{s}(u, \omega)<\varepsilon+\mu_{s}\left(u, E_{t}\right), \quad \mu(u, \omega \backslash K)<\varepsilon+\mu_{s}\left(u, E_{t}\right) .
$$

Fix $\delta>0$ and set

$$
\begin{aligned}
& \mathcal{X}^{\delta}:=\left\{B_{\rho}(x):\right. x \in E_{t}, \rho<\delta, \overline{B_{\rho}(x)} \subset \omega, \mu_{s}\left(u, \partial B_{\rho}(x)\right)=0, \\
& u \in W^{1, \alpha}\left(\partial B_{\rho}(x) ; \mathbb{R}^{d}\right), \\
&\left.\mathbf{m}\left(u, B_{\rho}(x)\right) \leqslant \int_{B_{\rho}(x)} f(y, \nabla u(y)) d y+t\left|B_{\rho}(x)\right|\right\}, \\
& \mathcal{Y}^{\delta}:=\left\{B_{\rho}(x): \rho<\delta, \overline{B_{\rho}(x)} \subset \omega \backslash K, \mu_{s}\left(u, \partial B_{\rho}(x)\right)=0\right\} .
\end{aligned}
$$


The balls of $\mathcal{X}^{\delta}$ and $\mathcal{Y}^{\delta}$ form a fine cover of $\omega$, and so Besicovitch Covering Theorem yields a countable subcover

$$
\omega=N \cup\left(\bigcup_{i=1}^{+\infty} B_{i}^{\mathcal{Y}}\right) \cup\left(\bigcup_{i=1}^{+\infty} B_{i}^{\mathcal{X}}\right)
$$

with $\mu(u, N)=0$, so that the balls $B_{i}^{\mathcal{Y}} \in \mathcal{Y}^{\delta}$ and $B_{i}^{\mathcal{X}} \in \mathcal{X}^{\delta}$ are all disjoint. By (3.13) we may take $n$ large enough such that

$$
\mu\left(u,\left[N \cup\left(\bigcup_{i} B_{i}^{\mathcal{Y}}\right) \cup\left(\bigcup_{i>n} B_{i}^{\mathcal{X}}\right)\right]\right)<\varepsilon+\mu_{s}\left(u, E_{t}\right),
$$

and for all $i \leqslant n$ choose $v_{i} \in W^{1, \beta}\left(B_{i}^{\mathcal{X}}\right)$ such that $v_{i}-u \in W_{0}^{1, \alpha}\left(B_{i}^{\mathcal{X}}\right)$ and

$$
F\left(v_{i}, B_{i}^{\mathcal{X}}\right) \leqslant \mathbf{m}\left(u, B_{i}^{\mathcal{X}}\right)+\delta\left|B_{i}^{\mathcal{X}}\right|
$$

Due to the choice of $\mathcal{X}^{\delta}$ and by (H3) we have

$$
\begin{aligned}
\int_{B_{i}^{\mathcal{X}}}\left|D v_{i}\right|^{\alpha} d x & \leqslant \int_{B_{i}^{\mathcal{X}}} f\left(x, \nabla v_{i}(x)\right) d x \leqslant \mathbf{m}\left(u, B_{i}^{\mathcal{X}}\right)+\delta\left|B_{i}^{\mathcal{X}}\right| \\
& \leqslant \int_{B_{i}^{\mathcal{X}}} f(x, \nabla u(x)) d x+(\delta+t)\left|B_{i}^{\mathcal{X}}\right|,
\end{aligned}
$$

and in view of the boundary assumption on $v_{i}$ and Poincaré inequality, using again (H3),

$$
\left\|v_{i}-u\right\|_{L^{\alpha}\left(B_{i}^{\mathcal{X}}\right)}^{\alpha} \leqslant C \delta^{\alpha}\left(\int_{B_{i}^{\mathcal{X}}} f(x, \nabla u(x)) d x+(\delta+t)\left|B_{i}^{\mathcal{X}}\right|\right),
$$

where we have invoked the fact that the radius of $B_{i}^{\mathcal{X}}$ is less than $\delta$. Set

$$
u_{\delta}(x):= \begin{cases}v_{i}(x) & \text { if } x \in B_{i}^{\mathcal{X}}, i \leqslant n \\ u(x) & \text { otherwise. }\end{cases}
$$

The previous inequality yields

$$
\left\|u_{\delta}-u\right\|_{L^{\alpha}(\omega)}^{\alpha} \leqslant C \delta^{\alpha}\left(\int_{\omega} f(x, \nabla u(x)) d x+|\omega|\right),
$$

thus $u_{\delta} \rightarrow u$ in $L^{\alpha}\left(\omega ; \mathbb{R}^{d}\right)$.

By Lemma 3.7, (3.14), and (3.15), we have

$$
\mathcal{F}\left(u_{\delta}, \omega\right)=\mathcal{F}\left(u, \omega \backslash \bigcup_{i \leqslant n} \overline{B_{i}^{\mathcal{X}}}\right)+\sum_{i \leqslant n} \mathcal{F}\left(u_{\delta}, B_{i}^{\mathcal{X}}\right) \leqslant \varepsilon+\mu_{s}\left(u, E_{t}\right)+\sum_{i \leqslant n} \mathcal{F}\left(v_{i}, B_{i}^{\mathcal{X}}\right)
$$


Since $v_{i} \in W^{1, \beta}\left(B_{i}^{\mathcal{X}} ; \mathbb{R}^{d}\right)$, by (2.5), (3.16), and in light of the choice of $\mathcal{X}^{\delta}$,

$$
\begin{aligned}
\mathcal{F}\left(u_{\delta}, \omega\right) & \leqslant \varepsilon+\mu_{s}\left(u, E_{t}\right)+\sum_{i \leqslant n} \mathbf{m}\left(u, B_{i}^{\mathcal{X}}\right)+\delta\left|\bigcup_{i \leqslant n} B_{i}^{\mathcal{X}}\right| \\
& \leqslant \varepsilon+\mu_{s}\left(u, E_{t}\right)+\int_{\omega} f(x, \nabla u(x)) d x+(\delta+t)|\omega| .
\end{aligned}
$$

The $L^{1}$ lower semicontinuity of $\mathcal{F}(\cdot, \omega)$ now yields

$$
\mathcal{F}(u, \omega) \leqslant \liminf _{\delta \rightarrow 0} \mathcal{F}\left(u_{\delta}, \omega\right) \leqslant \varepsilon+\mu_{s}\left(u, E_{t}\right)+\int_{\omega} f(x, \nabla u(x)) d x+t|\omega|,
$$

so letting $\omega \searrow E_{t}$ and then $\varepsilon \rightarrow 0$ we get

$$
\mu\left(u, E_{t}\right) \leqslant \mu_{s}\left(u, E_{t}\right)+t\left|E_{t}\right|+\int_{E_{t}} f(x, \nabla u(x)) d x .
$$

Since $\left|\Omega^{\prime} \backslash E_{t}\right|=0$ this implies

$$
\mathcal{F}\left(u, \Omega^{\prime}\right) \leqslant \mu\left(u, E_{t}\right)+\mu_{s}\left(u, \Omega^{\prime}\right) \leqslant 2 \mu_{s}\left(u, \Omega^{\prime}\right)+t\left|\Omega^{\prime}\right|+\int_{\Omega^{\prime}} f(x, \nabla u(x)) d x .
$$

Now let $x_{0}$ be a Lebesgue point for $f(\cdot, \nabla u(\cdot))$ such that

$$
\lim _{\rho \rightarrow 0} \frac{\mu_{s}\left(u, B_{\rho}\left(x_{0}\right)\right)}{\rho^{N}}=0
$$

taking $\Omega^{\prime}:=B_{\rho}\left(x_{0}\right)$ in the previous inequality, dividing through by $\left|B_{\rho}\left(x_{0}\right)\right|$, and letting $\rho \rightarrow 0$ we obtain

$$
\limsup _{\rho \rightarrow 0} \mathcal{F}_{\text {av }}\left(u, B_{\rho}\left(x_{0}\right)\right) \leqslant t+f\left(x_{0}, \nabla u\left(x_{0}\right)\right),
$$

and by (3.17) the result now follows by the arbitrariness of $t$.

We are now in position to prove Theorem 1.1.

Proof of Theorem 1.1. - The statement of Theorem 1.1 is now easily asserted by appealing to Proposition 3.3, Proposition 3.10, and to the fact that (see Theorem 2.4)

$$
\int_{A} f(x, \nabla u) d x \leqslant \mathcal{F}(u, A)
$$

for all $A \subset \Omega$.

In the remaining of this section we show that, near $x_{0} \in \Omega, \mathcal{F}(u, \cdot)$ may be determined by solving a Dirichlet problem. Precisely, 
THEOREM 3.11. - If $\mathcal{F}(u, \Omega)<+\infty$ then for $\mu(u, \cdot)$-a.e. $x_{0} \in \Omega$

$$
\lim _{\rho \rightarrow 0} \frac{\mathbf{m}\left(u, B_{\rho}\left(x_{0}\right)\right)}{\mathcal{F}\left(u, B_{\rho}\left(x_{0}\right)\right)}=1 .
$$

We first prove an auxiliary result.

Proposition 3.12. - If $\mathcal{F}(u, \Omega)<+\infty$ and if $B$ is an uplift ball for $u$ then

$$
\mathcal{F}(u, B) \geqslant \mathbf{m}(u, B) .
$$

Proof. - By the definition of uplift ball we have that $u \in W^{1, \alpha}\left(\partial B ; \mathbb{R}^{d}\right)$, and consider a sequence $\left\{u_{n}\right\}$ satisfying the conditions of Definition 3.5(ii). Set

$$
\bar{u}:=\mathrm{E}_{B} u, \quad w_{n}:=\mathrm{E}_{B}\left(u_{n}-u\right),
$$

so that $\bar{u}, w_{n} \in W^{1, \beta}(B), \bar{u}=u$ on $\partial B$. Since $u_{n} \rightarrow u$ in $W^{1, \alpha}(\partial B)$, by Lemma 2.1

$$
w_{n} \rightarrow 0 \quad \text { in } W^{1, \beta}(B) .
$$

Define for all $w \in W^{1, \beta}(B)$

$$
h(w):=\inf \left\{F(v, B): v \in W^{1, \beta}(B), v=\bar{u}+w \text { on } \partial B\right\},
$$

and remark that

$$
h(0)=\mathbf{m}(u, B), \quad h\left(w_{n}\right)=\mathbf{m}\left(u_{n}, B\right) .
$$

In order to show that $h$ is lower semicontinuous at 0 with respect to the strong topology of $W^{1, \beta}\left(B ; \mathbb{R}^{d}\right)$, we prove that it is convex and that it is locally bounded from above at 0 . To establish convexity we fix $w_{1}, w_{2} \in W^{1, \beta}\left(B ; \mathbb{R}^{d}\right), 0<t<1, \varepsilon>0$, and choose $v_{1}, v_{2} \in W^{1, \beta}\left(B ; \mathbb{R}^{d}\right)$ such that

$$
v_{i}=\bar{u}+w_{i} \quad \text { on } \partial B, \quad F\left(v_{i}, B\right) \leqslant h\left(w_{i}\right)+\varepsilon .
$$

Then $t v_{1}+(1-t) v_{2}=\bar{u}+t w_{1}+(1-t) w_{2}$ on $\partial B$, and by the convexity of $f$

$$
\begin{aligned}
h\left(t w_{1}+(1-t) w_{2}\right) & \leqslant F\left(t v_{1}+(1-t) v_{2}, B\right) \leqslant t F\left(v_{1}, B\right)+(1-t) F\left(v_{2}, B\right) \\
& \leqslant t h\left(w_{1}\right)+(1-t) h\left(w_{2}\right)+2 \varepsilon .
\end{aligned}
$$

It suffices now to let $\varepsilon \rightarrow 0$.

On the other hand, for all $w \in W^{1, \beta}\left(B ; \mathbb{R}^{d}\right)$

$$
h(w) \leqslant F(\bar{u}+w, B) \leqslant C\left(|B|+\|\nabla(\bar{u}+w)\|_{L^{\beta}(B)}^{\beta}\right) \leqslant C\left(|B|+\|\bar{u}+w\|_{W^{1, \beta}(B)}^{\beta}\right),
$$

from which we conclude that $h$ is bounded from above in the unit ball of $W^{1, \beta}\left(B ; \mathbb{R}^{d}\right)$.

In view of Lemma 3.4(c), (3.18), and (3.19) we now have

$$
\mathcal{F}(u, B)=\lim _{n \rightarrow+\infty} F\left(u_{n}, B\right) \geqslant \liminf _{n \rightarrow+\infty} \mathbf{m}\left(u_{n}, B\right)=\liminf _{n \rightarrow+\infty} h\left(w_{n}\right) \geqslant h(0)=\mathbf{m}(u, B) .
$$


Proof of Theorem 3.11. - By Proposition 3.12 we have

$$
\limsup _{\rho \rightarrow 0} \frac{\mathbf{m}\left(u, B_{\rho}\left(x_{0}\right)\right)}{\mathcal{F}\left(u, B_{\rho}\left(x_{0}\right)\right)} \leqslant 1 .
$$

For all $t>0$ set

$$
G_{t}:=\left\{B_{\rho} \subset \Omega: B_{\rho} \text { uplift ball, } \mathcal{F}\left(u, B_{\rho}\right)>\mathbf{m}\left(u, B_{\rho}\right)+t \mathcal{F}\left(u, B_{\rho}\right)\right\}
$$

and

$$
E_{t}:=\left\{x \in \Omega: \exists \rho_{h} \rightarrow 0 \text { with } B_{\rho_{h}}(x) \in G_{t}\right\} .
$$

We claim that $\mu\left(u, E_{t}\right)=0$, therefore establishing the converse of (3.20). Indeed, for every $x_{0} \in \Omega \backslash E_{t}$ there exists $\delta>0$ such that $B_{\rho}\left(x_{0}\right) \notin G_{t}$ for all $0<\rho<\delta$. This implies $(1-t) \mathcal{F}\left(u, B_{\rho}\left(x_{0}\right)\right) \leqslant \mathbf{m}\left(u, B_{\rho}\left(x_{0}\right)\right)$, hence

$$
\liminf _{\rho \rightarrow 0} \frac{\mathbf{m}\left(u, B_{\rho}\left(x_{0}\right)\right)}{\mathcal{F}\left(u, B_{\rho}\left(x_{0}\right)\right)} \geqslant 1-t
$$

If $\mu\left(u, E_{t}\right)=0$ then we conclude that (3.21) holds $\mu(u, \cdot)$-a.e. in $\Omega$. The arbitrariness of $t$ yields

$$
\liminf _{\rho \rightarrow 0} \frac{\mathbf{m}\left(u, B_{\rho}\left(x_{0}\right)\right)}{\mathcal{F}\left(u, B_{\rho}\left(x_{0}\right)\right)} \geqslant 1 .
$$

We now establish the claim. Note that at this point there is no guarantee that $E_{t}$ is even measurable, so we must proceed with care. Let $\mu^{*}(u, \cdot)$ be the Borel regular outer measure associated with $\mu(u, \cdot)$. Suppose, by contradiction, that $\mu^{*}\left(u, E_{t}\right)>\gamma$ for some $\gamma>0$. For all $\delta>0$ we define

$$
\mathcal{X}^{\delta}:=\left\{B_{\rho}(x): x \in E_{t}, \rho<\delta, B_{\rho}(x) \in G_{t}\right\} .
$$

This set forms a fine covering of $E_{t}$, so by Besicovitch Covering Theorem we may find a countable subcover such that

$$
E_{t} \subset N \cup\left(\bigcup_{i=1}^{+\infty} B_{i}^{\delta}\right),
$$

with $\mu(u, N)=0$ and for suitable mutually disjoint balls $B_{i}^{\delta} \in \mathcal{X}^{\delta}$. As $\mu(u, \Omega)<+\infty$, we may find $n_{\delta}$ large enough such that

$$
\mu\left(u, N \cup\left(\bigcup_{i>n_{\delta}} B_{i}^{\delta}\right)\right)<\frac{\gamma}{2},
$$

and thus

$$
\mu\left(u, \bigcup_{i \leqslant n_{\delta}} B_{i}^{\delta}\right)>\mu^{*}\left(u, E_{t}\right)-\frac{\gamma}{2}>\frac{\gamma}{2}
$$


For all $i \leqslant n_{\delta}$ choose $v_{i}^{\delta} \in W^{1, \beta}\left(B_{i}^{\delta} ; \mathbb{R}^{d}\right)$ such that $v_{i}^{\delta}-u \in W_{0}^{1, \alpha}\left(B_{i}^{\delta} ; \mathbb{R}^{d}\right)$ and

$$
F\left(v_{i}^{\delta}, B_{i}^{\delta}\right) \leqslant \mathbf{m}\left(u, B_{i}^{\delta}\right)+\frac{\delta}{n_{\delta}} .
$$

By (H3) and Proposition 3.12 we then get

$$
\int_{B_{i}^{\delta}}\left|\nabla v_{i}^{\delta}\right|^{\alpha} d x \leqslant F\left(v_{i}^{\delta}, B_{i}^{\delta}\right) \leqslant \mathbf{m}\left(u, B_{i}^{\delta}\right)+\frac{\delta}{n_{\delta}} \leqslant \mathcal{F}\left(u, B_{i}^{\delta}\right)+\frac{\delta}{n_{\delta}},
$$

hence

$$
\sum_{i \leqslant n} \int_{B_{i}^{\delta}}\left|\nabla v_{i}^{\delta}\right|^{\alpha} d x \leqslant \mathcal{F}(u, \Omega)+\delta<+\infty .
$$

Poincaré inequality now implies that

$$
\sum_{i \leqslant n_{\delta}}\left\|v_{i}^{\delta}-u\right\|_{L^{\alpha}\left(B_{i}^{\mathcal{X}}\right)}^{\alpha} \leqslant C \delta^{\alpha}
$$

where we used the fact that the radii of the balls $B_{i}^{\delta}$ are less than $\delta$. We define

$$
u_{\delta}(x):= \begin{cases}v_{i}^{\delta}(x) & \text { if } x \in B_{i}^{\delta}, i \leqslant n_{\delta} \\ u(x) & \text { otherwise. }\end{cases}
$$

Clearly $u_{\delta} \rightarrow u$ in $L^{\alpha}\left(U_{\delta_{0}}\right)$, and by our choice of the functions $v_{i}^{\delta}$ and by definition of the set $E_{t}$ we deduce that

$$
\begin{aligned}
\mathcal{F}\left(u_{\delta}, \Omega\right) & =\mathcal{F}\left(u, \Omega \backslash \bigcup_{i \leqslant n_{\delta}} B_{i}^{\delta}\right)+\sum_{i \leqslant n_{\delta}} F\left(v_{i}^{\delta}, B_{i}^{\delta}\right) \\
& \leqslant \mathcal{F}\left(u, \Omega \backslash \bigcup_{i \leqslant n_{\delta}} B_{i}^{\delta}\right)+\sum_{i \leqslant n_{\delta}} \mathbf{m}\left(u, B_{i}^{\delta}\right)+\delta \\
& \leqslant \mathcal{F}\left(u, \Omega \backslash \bigcup_{i \leqslant n_{\delta}} B_{i}^{\delta}\right)+\sum_{i \leqslant n_{\delta}}\left[\mathcal{F}\left(u, B_{i}^{\delta}\right)-t \mu\left(u, B_{i}^{\delta}\right)\right]+\delta \\
& \leqslant \mathcal{F}(u, \Omega)-t \mu\left(u, \bigcup_{i \leqslant n_{\delta}} B_{i}^{\delta}\right)+\delta \\
& \leqslant \mathcal{F}(u, \Omega)-\frac{t \gamma}{2}+\delta .
\end{aligned}
$$

By the $L^{1}$ lower semicontinuity of $\mathcal{F}$, as $\delta$ tends to 0 we obtain

$$
\mathcal{F}(u, \Omega) \leqslant \mathcal{F}(u, \Omega)-\frac{t \gamma}{2},
$$

which is impossible since $t \gamma / 2>0$ and $\mathcal{F}(u, \Omega)<+\infty$. We conclude that $\mu^{*}\left(u, E_{t}\right)$ $=0$. 
Remark 3.13. - If $\mathcal{F}(u, \Omega)<+\infty$ then

$$
f(x, \nabla u(x))=\lim _{\rho \rightarrow 0} \frac{\mathcal{F}\left(u, B_{\rho}(x)\right)}{\left|B_{\rho}(x)\right|}=\lim _{\rho \rightarrow 0} \frac{\mathbf{m}\left(u, B_{\rho}(x) r\right)}{\left|B_{\rho}(x)\right|} \text { for } \mathcal{L}^{N} \text {-a.e. } x .
$$

Indeed, if $f(x, \nabla u(x))>0$ then this follows from Theorems 1.1 and 3.11, and if $f(x, \nabla u(x))=0$ then it suffices to use Proposition 3.12 to deduce that

$$
\limsup _{\rho \rightarrow 0} \frac{\mathbf{m}\left(u, B_{\rho}(x)\right)}{\left|B_{\rho}(x)\right|} \leqslant \lim _{\rho \rightarrow 0} \frac{\mathcal{F}\left(u, B_{\rho}(x)\right)}{\left|B_{\rho}(x)\right|}=f(x, \nabla u(x))
$$

where the last equality is asserted by Theorem 1.1. Moreover

$$
\mu_{s}(u,\{x\})=\lim _{\rho \rightarrow 0} \mathbf{m}\left(u, B_{\rho}(x)\right) .
$$

Indeed, if $\mu_{s}(\{x\})=0$ then this equality is a direct consequence of Proposition 3.12, while if $\mu_{s}(\{x\})>0$ then Theorem 3.11 yields

$$
1=\lim _{\rho \rightarrow 0} \frac{\mathbf{m}\left(u, B_{\rho}\right)}{\mu\left(u, B_{\rho}\right)}=\lim _{\rho \rightarrow 0} \frac{\mathbf{m}\left(u, B_{\rho}(x)\right)}{\mu_{s}(u,\{x\})} .
$$

\section{Remarks on the Lavrentiev phenomenon}

In this section we prove Proposition 1.2 and Theorem 1.3.

Proof of Proposition 1.2. - By Theorem 1.1 there exists a nonnegative Radon measure $\mu(u, \cdot)$, singular with respect to $\mathcal{L}^{N}\llcorner\Omega$, such that

$$
\mathcal{F}(u, B)=\int_{B} f(x, \nabla u) d x+\mu_{s}(u, B)
$$

for every open set $B \subset A$. Condition (1.4) implies that for every $x \in A$ there exists an open neighborhood $U$ of $x$ such that $\mu_{s}(u, U)=0$. As $\mu_{s}(u, \cdot)$ is a nonnegative measure, we deduce that it vanishes on every subset of $A$. The conclusion follows now from (4.1).

Remark 4.1. - It can be easily verified that condition (1.4) is equivalent to assuming that there exists a sequence $\left\{u_{n}\right\} \subset W^{1, \beta}\left(B ; \mathbb{R}^{d}\right)$ such that

$$
u_{n} \rightarrow u \quad \text { in } W^{1, \alpha}\left(B ; \mathbb{R}^{d}\right), \quad F\left(u_{n}, B\right) \rightarrow F(u, B) .
$$

That (1.4) implies (4.2) follows immediately from the definition of $\mathcal{F}(u, B)$ : it suffices to choose any good sequence for $u$ in $B$ (see (2.4)). Conversely, by Ioffe's Theorem (see Theorem 2.4) we have that $\mathcal{F}\left(u, B_{\rho(x)}(x)\right) \geqslant F\left(u, B_{\rho(x)}(x)\right)$, while (4.2) yields the converse inequality; hence

$$
\mathcal{F}\left(u, B_{\rho(x)}(x)\right)=F\left(u, B_{\rho(x)}(x)\right) \quad \text { for all } x \in \Omega .
$$


Theorem 1.3 follows immediately from Proposition 1.2, Remark 4.1, and the lemma below.

LEMMA 4.2. - If $f$ is a Carathéodory function satisfying (1.5) and (1.6) then there exists a sequence $\left\{u_{n}\right\}$ such that

$$
u_{n} \in C_{0}^{\infty}(\Omega), \quad u_{n} \rightarrow u \quad \text { in } L_{\mathrm{loc}}^{1}(\Omega), \quad f\left(x, \nabla u_{n}\right) \rightarrow f(x, \nabla u) \quad \text { in } L_{\mathrm{loc}}^{1}(\Omega) .
$$

Moreover for every $x_{0} \in \Omega$ and for almost all $\rho<\operatorname{dist}\left(x_{0}, \partial \Omega\right)$

$$
u_{n} \rightarrow u \quad \text { strongly in } W^{1, \alpha}\left(B_{\rho}\left(x_{0}\right) ; \mathbb{R}^{d}\right)
$$

and there exists a subsequence (depending on $x_{0}$ ) such that

$$
u_{n} \rightarrow u \quad \text { strongly in } W^{1, \alpha}\left(\partial B_{\rho}\left(x_{0}\right) ; \mathbb{R}^{d}\right) .
$$

Proof. - Let $\rho(x)$ be a standard symmetric mollifier with support in $B_{1}(0)$, for every $n \in \mathbb{N}$ set $\rho_{n}(x):=n^{N} \rho(n x)$, and set

$$
u_{n}:=\bar{u}_{n} * \rho_{n}, \quad \bar{u}_{n}(x):= \begin{cases}u(x) & \text { if } \operatorname{dist}(x, \partial \Omega)>1 / n \\ 0 & \text { otherwise }\end{cases}
$$

Clearly $u_{n} \in C_{0}^{\infty}\left(\Omega ; \mathbb{R}^{d}\right), u_{n} \rightarrow u$ in $W_{\text {loc }}^{1, \alpha}(\Omega)$, thus $u_{n} \rightarrow u$ strongly in $W^{1, \alpha}\left(B_{r}\left(x_{0}\right) ; \mathbb{R}^{d}\right)$ for any ball $B_{r}\left(x_{0}\right)$ with $x_{0} \in \Omega$ and $r<\operatorname{dist}\left(x_{0}, \partial \Omega\right)$. Since for a fixed $B_{\rho}\left(x_{0}\right) \Subset \Omega$ and by Fubini's Theorem

$$
\begin{aligned}
\left\|u-u_{n}\right\|_{W^{1, \alpha}\left(B_{\rho}\right)}^{\alpha} & =\int_{0}^{\rho} \int_{\partial B_{r}\left(x_{0}\right)}\left[\left|u-u_{n}\right|^{\alpha}+\left|\nabla u-\nabla u_{n}\right|^{\alpha}\right] d H^{N-1} d r \\
& =\int_{0}^{\rho}\left\|u-u_{n}\right\|_{W^{1, \alpha}\left(\partial B_{r}\left(x_{0}\right)\right)}^{\alpha} d r,
\end{aligned}
$$

by Egoroff's Theorem we have that, up to a subsequence, also $u_{n} \rightarrow u$ strongly in $W^{1, \alpha}\left(\partial B_{r}\left(x_{0}\right) ; \mathbb{R}^{d}\right)$ for almost $r<\rho$. A diagonalization argument now yields a subsequence for which (4.3) holds for almost all $\rho<\operatorname{dist}\left(x_{0}, \partial \Omega\right)$. We only have to show that

$$
f\left(x, \nabla u_{n}\right) \rightarrow f(x, \nabla u) \quad \text { in } L_{\mathrm{loc}}^{1}(\Omega) .
$$

Fix $A \Subset \Omega$, and let $\operatorname{dist}(A, \partial \Omega) \geqslant 2 \varepsilon_{0}$; without loss of generality we may always take $n>1 / \varepsilon_{0}$, thus in $A$ we have $D u_{n}=D u * \rho_{n}$. Set $A^{\prime}:=\left\{x \in \Omega\right.$ : $\left.\operatorname{dist}(x, \partial \Omega) \geqslant \varepsilon_{0}\right\}$, and define

$$
g(x):=f(x, D u(x)), \quad p_{n}(x)=\min \{p(y):|x-y| \leqslant 1 / n\}, \quad \varphi_{n}(x, z)=|z|^{p_{n}(x)} .
$$

Clearly $\varphi_{n}$ is a Carathéodory function, $\varphi(x, \cdot)$ is convex for all $x \in \Omega$, and if $|x-y|<$ $1 / n$ then $p_{n}(x) \leqslant p(y)$ and so

$$
\varphi_{n}(x, z)=|z|^{p_{n}(x)} \leqslant 1+|z|^{p(y)} \leqslant 1+f(y, z) .
$$


In view of this inequality and by Jensen's inequality, if $x \in A$ then

$$
\begin{aligned}
\varphi_{n}\left(x, \nabla u_{n}(x)\right) & =\varphi_{n}\left(x, \int_{B(x, 1 / n)} \nabla u(y) \rho_{n}(x-y) d y\right) \\
& \leqslant \int_{B(x, 1 / n)} \varphi_{n}(x, \nabla u(y)) \rho_{n}(x-y) d y \\
& \leqslant \int_{B(x, 1 / n)}[1+f(y, \nabla u(y))] \rho_{n}(x-y) d y \\
& =1+\left(g * \rho_{n}\right)(x) .
\end{aligned}
$$

Now remark that if $x \in A$, then using the fact that $u \in W_{\mathrm{loc}}^{1, \alpha}$ (see (1.5)), we have

$$
\begin{aligned}
\left|\nabla u_{n}(x)\right| & =\left|\int_{A^{\prime}} \nabla u(y) \rho_{n}(x-y) d y\right| \\
& \leqslant C(\alpha)\|\nabla u\|_{L^{\alpha}\left(A^{\prime}\right)} n^{N}\left|\int_{A^{\prime}} \rho^{\alpha^{\prime}}(n(x-y)) d y\right|^{1 / \alpha^{\prime}} \\
& \leqslant C(\alpha)\|\nabla u\|_{L^{\alpha}\left(A^{\prime}\right)} n^{N} n^{-N / \alpha^{\prime}}\left|\int_{\mathbb{R}^{N}} \rho^{\alpha^{\prime}}(y) d y\right|^{1 / \alpha^{\prime}} \\
& =C^{\prime}\|\nabla u\|_{L^{\alpha}\left(A^{\prime}\right)} n^{N / \alpha}
\end{aligned}
$$

where $C^{\prime}=C^{\prime}(\alpha)$ is a positive constant. By (1.5) we deduce that

$$
\begin{aligned}
\frac{1}{C} f\left(x, \nabla u_{n}(x)\right)-1 & \leqslant\left|\nabla u_{n}(x)\right|^{p(x)}=\left|\nabla u_{n}(x)\right|^{p(x)-p_{n}(x)} \varphi_{n}\left(x, \nabla u_{n}(x)\right) \\
& \leqslant\left(1+C^{\prime \beta-\alpha}\|\nabla u\|_{L^{\alpha}\left(A^{\prime}\right)}^{\beta-\alpha}\right)\left(n^{p(x)-p_{n}(x)}\right)^{N / \alpha} \varphi_{n}\left(x, \nabla u_{n}(x)\right) \\
& \leqslant C \mathrm{e}^{N \gamma / \alpha} \varphi_{n}\left(x, \nabla u_{n}(x)\right),
\end{aligned}
$$

where we have used the fact that

$$
0 \leqslant p(x)-p_{n}(x) \leqslant \gamma / \log n
$$

so that

$$
\left(n^{p(x)-p_{n}(x)}\right)^{N / \alpha} \leqslant \mathrm{e}^{N \gamma / \alpha} .
$$

In view of (4.5) we now have for all $x \in A$

$$
0 \leqslant f\left(x, \nabla u_{n}(x)\right) \leqslant C\left(1+\left(g * \rho_{n}\right)(x)\right) .
$$

Since $\nabla u_{n} \rightarrow \nabla u$ a.e. in $A$, by the continuity of $f(x, \cdot)$ and Fatou's Lemma we have

$$
\liminf _{n \rightarrow+\infty} \int_{A} f\left(x, \nabla u_{n}(x)\right) d x \geqslant \int_{A} f(x, \nabla u(x)) d x
$$


Moreover, once more by Fatou's Lemma and using the fact that $g * \rho_{n} \rightarrow g$ in $L^{1}(A)$,

$$
\begin{array}{rl}
\int_{A} & C(1+g(x))-\limsup _{n \rightarrow+\infty} \int_{A} f\left(x, \nabla u_{n}(x)\right) d x \\
= & \liminf _{n \rightarrow+\infty} \int_{A}\left[C\left(1+\left(g * \rho_{n}\right)(x)\right)-f\left(x, \nabla u_{n}(x)\right)\right] d x \\
\geqslant & \int_{A}[C(1+g(x))-f(x, \nabla u(x))] d x,
\end{array}
$$

and we conclude that

$$
\limsup _{n \rightarrow+\infty} \int_{A} f\left(x, \nabla u_{n}(x)\right) d x \leqslant \int_{A} f(x, \nabla u(x)) d x .
$$

This, together with (4.6), yields the convergence of $f\left(\cdot, \nabla u_{n}(\cdot)\right)$ to $f(\cdot, \nabla u(\cdot))$ in $L^{1}(A)$.

The last result of this section shows that under some additional assumptions on the convergence of the traces of approximating sequences, it is possible to impose Dirichlet boundary conditions on the admissible sequences without increasing the overall energy. Since a priori the infimum taken over the class of functions restricted under the Dirichlet boundary condition is greater than the infimum over the unconstrained class, we may interpret this result as a situation where the Lavrentiev phenomenon is avoided.

Proposition 4.3. - Let $\mathcal{F}(u, \Omega)<+\infty$, let $A \subset \Omega$ be an open set with Lipschitz boundary, and assume that $u_{n} \in W^{1, \beta}(A)$ satisfies

$$
u_{n} \rightarrow u \quad \text { weakly in } W^{1, \alpha}(A) \text { and weakly in } W^{1, \alpha}(\partial A),
$$

and

$$
\mathcal{F}(u, A)=\lim _{n \rightarrow+\infty} F\left(u_{n}, A\right) .
$$

Then there exists a sequence $v_{n} \in W^{1, \beta}(A)$ such that

$$
v_{n}=u \quad \text { on } \partial A, \quad v_{n} \rightarrow u \quad \text { weakly in } W^{1, \alpha}(A), \quad \mathcal{F}(u, A)=\lim _{n \rightarrow+\infty} F\left(v_{n}, A\right) .
$$

Moreover if the sequence $\left\{u_{n}\right\}$ converges to $u$ strongly in $W^{1, \alpha}(A)$ then so does $\left\{v_{n}\right\}$.

Proof. - Set $w_{n}=\mathrm{E}_{A}\left(u_{n}-u\right)$, so that by Lemma $2.1 w_{n} \rightarrow 0$ strongly in $W^{1, \beta}(A)$ and $w_{n}=u_{n}-u$ on $\partial A$. For $k \in \mathbb{N}$ let $\varepsilon=1 / k$ and define $h_{\varepsilon}: W^{1, \beta}(A) \rightarrow[0,+\infty]$ by

$$
h_{\varepsilon}(w):=\inf \left\{F(v, A): v \in W^{1, \beta}(A), v=u+w \text { on } \partial A,\|v-u\|_{L^{1}(A)}<\varepsilon\right\} .
$$

Since for $n \in \mathbb{N}$ sufficiently large

$$
u_{n}=u+w_{n} \text { on } \partial A \text { and }\left\|u_{n}-u\right\|_{L^{1}(A)}<\varepsilon \text {, }
$$


it follows that

$$
h_{\varepsilon}\left(w_{n}\right) \leqslant F\left(u_{n}, A\right) .
$$

A similar proof to that of Proposition 3.12 yields the convexity of $h_{\varepsilon}$. Also, let $C(\varepsilon)>0$ be such that

$$
\|w\|_{W^{1, \beta}(A)}<C(\varepsilon) \quad \Rightarrow \quad\|w\|_{L^{1}(A)}<\varepsilon / 2 .
$$

We claim that $h_{\varepsilon}$ is bounded in the ball in $W^{1, \beta}(A)$ of center zero and radius $C(\varepsilon)$. Indeed, as $u \in W^{1, \alpha}(\partial A)$ there exists $\bar{u} \in W^{1, \beta}(A)$ such that $u-\bar{u} \in W_{0}^{1, \alpha}(A)$, but then there exists $\varphi_{\varepsilon} \in C_{0}^{\infty}(A)$ such that $\left\|(u-\bar{u})-\varphi_{\varepsilon}\right\|_{W^{1, \alpha}(A)}<\varepsilon / 2$. Setting $u_{\varepsilon}:=\bar{u}+\varphi_{\varepsilon}$ we have $u_{\varepsilon} \in W^{1, \beta}(A), u_{\varepsilon}=u$ on $\partial A,\left\|u_{\varepsilon}-u\right\|_{W^{1, \alpha}(A)}<\varepsilon / 2$, and thus for all $w \in W^{1, \beta}(A)$ with $\|w\|_{W^{1, \beta}(A)}<C(\varepsilon)$, and in view of $(\mathrm{H} 3)$

$$
h_{\varepsilon}(w) \leqslant F\left(u_{\varepsilon}+w, A\right) \leqslant C\left(|A|+\left\|\left(u_{\varepsilon}+w\right)\right\|_{W^{1, \beta}(A)}^{\beta}\right) \leqslant C^{\prime}(\varepsilon)
$$

for some constant $C^{\prime}(\varepsilon)>0$. Therefore the convex function $h_{\varepsilon}$ is lower semicontinuous at the origin, and by (4.7) we have

$$
\mathcal{F}(u, A)=\lim _{n \rightarrow+\infty} F\left(u_{n}, A\right) \geqslant \lim _{n \rightarrow+\infty} h_{\varepsilon}\left(w_{n}\right) \geqslant h_{\varepsilon}(0),
$$

that is

$$
\mathcal{F}(u, A) \geqslant \inf \left\{F(v, A): v \in W^{1, \beta}(A), v=u \text { on } \partial A,\|v-u\|_{L^{1}(A)}<\varepsilon\right\} .
$$

In particular, there exists $v_{\varepsilon} \in W^{1, \beta}(A)$ such that $v_{\varepsilon}=u$ on $\partial A$ and

$$
\left\|v_{\varepsilon}-u\right\|_{L^{1}(A)}<\varepsilon, \quad F\left(v_{\varepsilon}, A\right) \leqslant \mathcal{F}(u, A)+\varepsilon
$$

and the result now follows by letting $\varepsilon \rightarrow 0$.

If $u_{n} \rightarrow u$ strongly in $W^{1, \alpha}(A)$ then one may replace the $L^{1}$ norm in the definition of $h_{\varepsilon}$ by the norm in $W^{1, \alpha}$, thus obtaining in (4.8) that $\left\|v_{\varepsilon}-u\right\|_{W^{1, \alpha}(A)}<\varepsilon$.

\section{A counterexample for Carathéodory quasiconvex integrands}

Here we present the proof of Proposition 1.4.

Recall that under (H1), (H3), by Theorem 3.1 in [15] if $u \in W^{1, \alpha}\left(\Omega ; \mathbb{R}^{d}\right)$ is such that $\mathcal{F}(u, \Omega)<+\infty$ then

$$
\mathcal{F}(u, A)=\int_{A} f^{u}(x) d x+\mu_{s}(u, A) \quad \text { for all } A \in \mathbb{A}(\Omega),
$$

where $\mu_{s}(u, \cdot)$ is a nonnegative finite Radon measure on $\Omega$, singular with respect to $\mathcal{L}^{N}\left\llcorner\Omega\right.$, and (see Theorem 3.2 in [6]) for a.e. $x_{0} \in \Omega$

$$
f^{u}\left(x_{0}\right)=\lim _{\rho \rightarrow 0} \frac{\mathbf{m}\left(u, B_{\rho}\left(x_{0}\right)\right)}{\left|B_{\rho}\left(x_{0}\right)\right|} .
$$


Here the limit is taken for $\rho \in \mathcal{E}\left(u, x_{0}\right)$, the set of good radii of the function $u$ at the point $x_{0} \in \Omega$ as defined in [6], Definition 3.4; it can be shown that almost all $r \in\left(0, \operatorname{dist}\left(x_{0}, \partial \Omega\right)\right)$ belong to $\mathcal{E}\left(u, x_{0}\right)$.

The following lemma may be found in [6], Lemma 3.5.

LEMMA 5.1. - Under assumptions (H1) and (H3), if $R \in \mathcal{R}\left(u, x_{0}\right)$ and if $\left\{u_{n}\right\} \subset$ $W^{1, p}\left(\Omega ; \mathbb{R}^{d}\right)$ is such that $u_{n} \rightarrow u$ weakly in $W^{1, p}\left(\partial B_{R}\left(x_{0}\right) ; \mathbb{R}^{d}\right)$ then

$$
\mathbf{m}\left(u, B_{R}\left(x_{0}\right)\right)=\lim _{n \rightarrow+\infty} \mathbf{m}\left(u_{n}, B_{R}(x)\right) .
$$

Next we simplify the representation formula for $f^{u}$ in the general quasiconvex context and in the case where $u$ is affine.

LEMMA 5.2. - If (H1) and (H3) hold, and if $u$ is affine, then for $\mathcal{L}^{N}$-a.e. $x_{0} \in \Omega$

$$
\begin{array}{r}
f^{u}\left(x_{0}\right)=\lim _{n \rightarrow+\infty}\left\{\inf _{B} f\left(x_{0}+\varepsilon_{n} y, \nabla w(y)\right) d y: w \in W^{1, \beta}\left(B ; \mathbb{R}^{d}\right),\right. \\
\left.w-u \in W_{0}^{1, \alpha}\left(B ; \mathbb{R}^{d}\right)\right\},
\end{array}
$$

where $B$ is the unit ball centered at the origin and $\left\{\varepsilon_{n}\right\} \subset \mathcal{E}\left(u, x_{0}\right)$ is any sequence of positive numbers converging to 0 .

Proof. - Consider $\left\{\varepsilon_{n}\right\} \subset \mathcal{E}\left(u, x_{0}\right)$ with $\varepsilon_{n} \rightarrow 0^{+}$. By (5.1) we have

$$
\begin{gathered}
f^{u}\left(x_{0}\right)=\lim _{n \rightarrow+\infty}\left\{\inf f_{B_{\varepsilon_{n}}\left(x_{0}\right)} f(x, \nabla v(x)) d x: v \in W^{1, \beta}\left(B_{\varepsilon_{n}}\left(x_{0}\right) ; \mathbb{R}^{d}\right),\right. \\
\left.v-u \in W_{0}^{1, \alpha}\left(B_{\varepsilon_{n}}\left(x_{0}\right) ; \mathbb{R}^{d}\right)\right\} \\
=\lim _{n \rightarrow+\infty}\left\{\inf _{B} f\left(x_{0}+\varepsilon_{n} y, \nabla v\left(x_{0}+\varepsilon_{n} y\right)\right) d y: v \in W^{1, \beta}\left(B_{\varepsilon_{n}}\left(x_{0}\right) ; \mathbb{R}^{d}\right),\right. \\
\left.v-u \in W_{0}^{1, \alpha}\left(B_{\varepsilon_{n}}\left(x_{0}\right) ; \mathbb{R}^{d}\right)\right\} \\
=\lim _{n \rightarrow+\infty}\left\{\inf _{B} f\left(x_{0}+\varepsilon_{n} y, \nabla w(y)\right) d y: w \in W^{1, \beta}\left(B ; \mathbb{R}^{d}\right),\right. \\
\left.w-u \in W_{0}^{1, \alpha}\left(B ; \mathbb{R}^{d}\right)\right\},
\end{gathered}
$$

where we used the substitution

$$
w(y):=\frac{v\left(x_{0}+\varepsilon_{n} y\right)-u\left(x_{0}\right)+\varepsilon_{n} u(0)}{\varepsilon_{n}}
$$

for $y \in B$, and we invoked the fact that $u$ is affine. 
Proof of Proposition 1.4. - Let $K, p$ and $f$ be as stated. We claim that for all $\delta \in(0,1)$ there exists $\theta_{0} \in(0,1)$ such that for all $\theta<\theta_{0}$ and for all $x_{0} \in \partial K$

$$
f^{\mathrm{id}}\left(x_{0}\right)<f\left(x_{0}, \mathbb{I}\right)-1+\delta
$$

Fix $x_{0} \in \partial K$ and let $\varepsilon \in(0,1)$. Since $0 \in \partial\left(K-x_{0}\right)$ we may find $y_{\varepsilon} \in B_{\varepsilon^{2}}(0)$ such that $y_{\varepsilon} \notin K-x_{0}$. Define $x_{\varepsilon}:=y_{\varepsilon} / \varepsilon$. Then $\left|x_{\varepsilon}\right|<\varepsilon$ and $x_{\varepsilon} \notin\left(K-x_{0}\right) / \varepsilon$. Denoting by $B$ the unit ball $B_{1}(0)$, we clearly have

$$
B\left(x_{\varepsilon}, 1-\left|x_{\varepsilon}\right|\right) \subset B, \quad\left|B \backslash B\left(x_{\varepsilon}, 1-\left|x_{\varepsilon}\right|\right)\right| \rightarrow 0 \quad \text { as } \varepsilon \rightarrow 0 .
$$

Since $\left(K-x_{0}\right) / \varepsilon$ is closed, there exists $r_{\varepsilon} \in(0,1)$ such that

$$
B\left(x_{\varepsilon}, r_{\varepsilon}\right) \subset B\left(x_{\varepsilon}, 1-\left|x_{\varepsilon}\right|\right) \backslash \frac{K-x_{0}}{\varepsilon},
$$

and we define in $B$ the function

$$
v_{\varepsilon}(x):= \begin{cases}x & \text { if } x \notin B\left(x_{\varepsilon}, 1-\left|x_{\varepsilon}\right|\right), \\ x_{\varepsilon}+\left(1-\left|x_{\varepsilon}\right|\right) \frac{x-x_{\varepsilon}}{\left|x-x_{\varepsilon}\right|} & \text { if } r_{\varepsilon}<\left|x-x_{\varepsilon}\right|<1-\left|x_{\varepsilon}\right|, \\ x_{\varepsilon}+\frac{1-\left|x_{\varepsilon}\right|}{r_{\varepsilon}}\left(x-x_{\varepsilon}\right) & \text { if }\left|x-x_{\varepsilon}\right|<r_{\varepsilon} .\end{cases}
$$

Clearly $v_{\varepsilon} \in W^{1, \infty}\left(B ; \mathbb{R}^{N}\right), v_{\varepsilon}=$ id on $\partial B$, and

$$
\begin{aligned}
f_{B}\left|\nabla v_{\varepsilon}\right|^{p} d x \leqslant & +\int_{B\left(x_{\varepsilon}, 1-\left|x_{\varepsilon}\right|\right) \backslash B\left(x_{\varepsilon}, r_{\varepsilon}\right)}\left|\frac{\mathbb{I}}{\left|x-x_{\varepsilon}\right|}-\frac{\left(x-x_{\varepsilon}\right) \otimes\left(x-x_{\varepsilon}\right)}{\left|x-x_{\varepsilon}\right|^{3}}\right|^{p} d x \\
& +\int_{B_{r_{\varepsilon}}\left(x_{\varepsilon}\right)}\left|\frac{1-\left|x_{\varepsilon}\right|}{r_{\varepsilon}}\right|^{p} d x \\
\leqslant & 1+C \int_{r_{\varepsilon}}^{1-\left|x_{\varepsilon}\right|} r^{N-1-p} d r+C r_{\varepsilon}^{N-p} \leqslant C_{0}
\end{aligned}
$$

where $C_{0}$ is a constant independent of $x_{0}$, and where we have used the fact that $p<N$. In view of Lemma 5.2, and choosing a sequence $\left\{\varepsilon_{n}\right\} \subset \mathcal{E}\left(\mathrm{id}, x_{0}\right)$ of positive numbers converging to 0 , we have

$$
\begin{aligned}
f^{\mathrm{id}}\left(x_{0}\right)= & \lim _{n \rightarrow+\infty}\left\{\operatorname { i n f } f _ { B } \left[\chi_{K}\left(x_{0}+\varepsilon_{n} x\right)|\operatorname{det} \nabla w(x)|\right.\right. \\
& \left.\left.\quad+\theta|\nabla w(x)|^{p}\right] d x: w \in W^{1, N}\left(B ; \mathbb{R}^{N}\right), w=\operatorname{id} \text { on } \partial B\right\} \\
\leqslant & \liminf _{n \rightarrow+\infty} \int_{B}\left[\chi \frac{K-x_{0}}{\varepsilon_{n}}(x)\left|\operatorname{det} \nabla v_{\varepsilon_{n}}(x)\right|+\theta\left|\nabla v_{\varepsilon_{n}}(x)\right|^{p}\right] d x \\
\leqslant & \liminf _{n \rightarrow+\infty} \frac{\left|B \backslash B\left(x_{\varepsilon_{n}}, 1-\left|x_{\varepsilon_{n}}\right|\right)\right|}{|B|}+\theta C_{0}=\theta C_{0},
\end{aligned}
$$


where we have used (5.3), (5.4), and the fact that $\operatorname{det} \nabla v_{\varepsilon_{n}}(x)=0$ if $r_{\varepsilon_{n}}<\left|x-x_{\varepsilon_{n}}\right|<$ $1-\left|x_{\varepsilon_{n}}\right|$. Since $f\left(x_{0}, \mathbb{I}\right) \geqslant 1$, we have now established the claim with $\theta_{0}=\delta / C_{0}$.

\section{Acknowledgements}

The research of E. Acerbi was partially supported by GNAFA-CNR under the Research Project "Modelli variazionali sotto ipotesi non standard - 2000".

The research of I. Fonseca was partially supported by the National Science Foundation under Grant No. DMS-9731957 and by the Center for Nonlinear Analysis (NSF Grant No. DMS-9803791). I. Fonseca also thanks the Universities of Parma and of Toulon et Var for their hospitality during the final stage of the paper.

The authors are indebted to the Max-Planck Institute in Leipzig for its support during the preparation of this work.

The authors are grateful to the referee for his/her very useful comments and for having corrected a previously faulty proof of Theorem 3.11.

\section{REFERENCES}

[1] E. Acerbi, G. Dal Maso, New lower semicontinuity results for polyconvex integrals, Cal. Var. 2 (1994) 329-337.

[2] E. Acerbi, N. Fusco, Semicontinuity problems in the calculus of variations, Arch. Rational Mech. Anal. 86 (1984) 125-145.

[3] J. Ball, Convexity conditions and existence theorems in nonlinear elasticity, Arch. Rational Mech. Anal. 63 (1977) 337-403.

[4] J.M. Ball, F. Murat, $W^{1, p}$ quasiconvexity and variational problems for multiple integrals, J. Funct. Anal. 58 (1984) 225-253.

[5] G. Bouchitté, I. Fonseca, G. Leoni, L. Mascarenhas, A global method for relaxation in $W^{1, p}$ and in $S B V_{p}$, To appear in Arch. Rational Mech. Anal.

[6] G. Bouchitté, I. Fonseca, J. Malý, The effective bulk energy of the relaxed energy of multiple integrals below the growth exponent, Proc. Roy. Soc. Edinburgh Sect. A 128 (1998) 463479.

[7] G. Bouchitté, I. Fonseca, L. Mascarenhas, A global method for relaxation, Arch. Rational Mech. Anal. 144 (1998) 1-46.

[8] L. Carbone, R. De Arcangelis, Further results on $\Gamma$-convergence and lower semicontinuity of integral functionals depending on vector-valued functions, Ricerche Mat. 39 (1990) 99129.

[9] P. Celada, G. Dal Maso, Further remarks on the lower semicontinuity of polyconvex integrals, Ann. Inst. Henri Poincaré Anal. Non Linéaire 11 (1994) 661-691.

[10] B. Dacorogna, Direct Methods in the Calculus of Variations, in: Applied Math. Sciences, Vol. 78, Springer-Verlag, 1989.

[11] B. Dacorogna, P. Marcellini, Semicontinuité pour des intégrandes polyconvexes sans continuité des determinants, C. R. Acad. Sci. Paris Sér. I Math. 311 (1990) 393-396.

[12] G. Dal Maso, C. Sbordone, Weak lower semicontinuity of polyconvex integrals: a borderline case, Math. Z. 218 (1995) 603-609.

[13] L.C. Evans, R.F. Gariepy, Measure Theory and Fine Properties of Functions, CRC Press, Boca Raton, 1992.

[14] X. Fan, D. Zhao, A class of De Giorgi type and Hölder continuity, Nonlinear Anal. TMA 36 (A) (1999) 295-318. 
[15] I. Fonseca, J. Malý, Relaxation of multiple integrals below the growth exponent, Ann. Inst. H. Poincaré Anal. Non Linéaire 14 (1997) 309-338.

[16] I. Fonseca, P. Marcellini, Relaxation of multiple integrals in subcritical Sobolev spaces, J. Geom. Anal. 7 (1997) 57-81.

[17] N. Fusco, J.E. Hutchinson, A direct proof for lower semicontinuity of polyconvex functionals, Manuscripta Math. 85 (1995) 35-50.

[18] W. Gangbo, On the weak lower semicontinuity of energies with polyconvex integrands, J. Math. Pures Appl. 73 (1994) 455-469.

[19] A.D. Ioffe, On lower semicontinuity of integral functionals I, SIAM J. Control Optim. 15 (1977) 521-538.

[20] R.D. James, S.J. Spector, The formation of filamentary voids in solids, J. Mech. Phys. Solids 39 (1991) 783-813.

[21] R.D. James, S.J. Spector, The formation of filamentary voids in solids, Proc. Roy. Soc. Edinburgh Sect. A 123 (1993) 681-691.

[22] J. Malý, Weak lower semicontinuity of polyconvex and quasiconvex integrals, Preprint, Vortragsreihe, Bonn, 1993.

[23] J. Malý, Lower semicontinuity of quasiconvex integrals, Manuscripta Math. 85 (1994) 419428.

[24] P. Marcellini, Approximation of quasiconvex functions, and lower semicontinuity of multiple integrals, Manuscripta Math. 51 (1985) 1-28.

[25] P. Marcellini, On the definition and the lower semicontinuity of certain quasiconvex integrals, Ann. Inst. H. Poincaré Anal. Non Linéaire 3 (1986) 391-409.

[26] C.B. Morrey, Multiple integrals in the Calculus of Variations, Springer, Berlin, 1966.

[27] S. Müller, S.J. Spector, An existence theory for nonlinear elasticity that allows for cavitation, Arch. Rational Mech. Anal. 131 (1995) 1-66.

[28] J. Sivaloganathan, On cavitation and degenerate cavitation under internal hydrostatic pressure, Roy. Soc. London Proc. Ser. A Math. Phys. Eng. Sci. 455 (1999) 3645-3664.

[29] V.V. Zhikov, On Lavrentiev's phenomenon, Russian J. Math. Phys. 3 (1995) 249-269.

[30] V.V. Zhikov, On some variational problems, Russian J. Math. Phys. 5 (1997) 105-116. 